\title{
META-MODELING OF OCCUPANCY VARIABLES AND ANALYSIS OF THEIR IMPACT ON ENERGY OUTCOMES OF OFFICE BUILDINGS
}

\author{
Qinpeng Wang, Godfried Augenbroe, Ji-Hyun Kim, Li Gu \\ Georgia Institute of Technology \\ 247 4th St NW, Atlanta, GA 30332, USA \\ Phone +1-404-372-8668, email: qpwang@ gatech.edu
}

\section{ABSTRACT}

Occupants interact with buildings in various ways via their presence (passive effects) and control actions (active effects). Therefore, understanding the influence of occupants is essential if we are to evaluate the performance of a building. In this paper, we model the mean profiles and variability of occupancy variables (presence and actions) separately. We will use a multi-variate Gaussian distribution to generate mean profiles of occupancy variables, while the variability will be represented by a multi-dimensional time series model, within a framework for a meta-analysis that synthesizes occupancy data gathered from a pool of buildings. We then discuss variants of occupancy models with respect to various outcomes of interest such as HVAC energy consumption and peak demand behavior via a sensitivity analysis. Results show that our approach is able to generate stochastic occupancy profiles, requiring minimum additional input from the energy modeler other than standard diversity profiles. Along with the meta-analysis, we enable the generalization of previous research results and statistical inferences to choose occupancy variables for future buildings. The sensitivity analysis shows that for aggregated building energy consumption, occupant presence has a smaller impact compared to lighting and appliance usage. Specifically, being accumulatively 55\% wrong with regard to presence, only translates to $2 \%$ error in aggregated cooling energy in July and 3.6\% error in heating energy in January. Such a finding redirects focus to the accurate estimation of lighting and appliance usage for a better prediction of aggregated energy consumption. Furthermore, it proves that accurate knowledge of the mean profiles is sufficient, that is, stochastic occupancy models do not play a significant role in the prediction of aggregated consumption in a conventional office building where the interaction between the operation of building systems and the spatial and temporal variability of occupancy is weak. When it comes to peak demand behavior, occupancy variability should be taken into account, as static profiles are not able to produce adequate estimates of power duration probabilities close to the power peak.

\section{KEY WORDS}

Occupancy representation; Stochastic occupancy modeling; Meta-analysis; Sensitivity analysis and ranking; Transfer function model

\section{INTRODUCTION}

The operation and energy use of buildings are highly dependent on the needs and behavior of occupants, through a complex relationship. Occupants, simply by being present in a building, participate in the heat balance of the building through their body heat. Furthermore, both occupants' intervention in the control of heating, ventilation and air conditioning systems, and their operation of lighting and appliances have an impact on building energy 
consumption. An inadequate representation of occupancy may undermine the credibility of our model findings in terms of the amount of energy conservation, mitigation of environmental pollutants and benefits of sustainable energy systems in buildings. In this paper, we denote the combination of the temporal and spatial presence, movement and actions of occupants as "occupancy" and variables representing occupancy in building energy models will be articulated in terms of "occupancy variables". In the remaining of this introductory chapter, we start with the problem statement that lies at the heart of this paper, followed by a thorough literature review of the current state-of-the-art occupancy modeling methods and efforts on characterizing the impact of variants of occupancy representation on various energy outcomes.

\subsection{Problem statement}

The goal of this paper is to guide a typical energy modeler through the modeling of occupancy variables when no long-term measurement data about the building of interest are available. In all cases, a typical energy modeler has access to some information relevant to the occupancy of the building that ranges from almost no information to very deep information, as depicted in Figure 1. On one end of the spectrum (Point A), we define "total ignorance", which corresponds to the case that the energy modeler has only the most rudimentary information about how the building intends to be used, which may be the normal case during preliminary design exploration. At the other end of the spectrum, we define "perfect knowledge", which occurs when the modeler has access to extensive measurement data in terms of occupancy variables. In most situations, one is somewhere between these extremes, which can be characterized as "intermediate knowledge". In those cases, the modeler has some information about typical ${ }^{1}$ building occupancy in part or as a whole, obtained through on-site audits (in case of retrofit) or tenant surveys (in case of new design). However, on-site audits and surveys generally will only convey a limited or even biased expectation of the typical occupancy of the building, while leaving out information about occupancy variability, in other words, the day-to-day fluctuations around the mean. Such information gap could be accounted for and supplemented with detailed data collection and analysis of similar existing buildings, i.e. having the same occupant organization type and employee demographics.

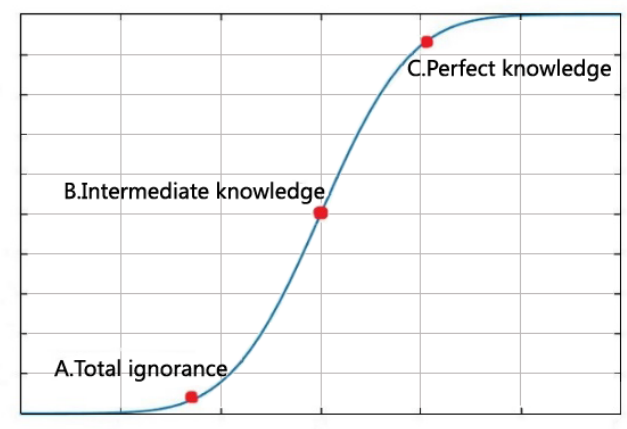

Information Level

Figure 1 Occupancy information available to the modeler (from low to high)

As such, it seems logical to conclude that a desirable modeling framework of occupancy tailored to the above problem statement should incorporate the following features:

- Supporting different strategies of occupancy representation, corresponding to the modeler's level of knowledge

- Accounting for a potentially biased and uncertain view of the modeler on typical building occupancy

\footnotetext{
1 "Typical' in the sense of mean profiles of occupancy.
} 
- Being able to extract and learn the characteristics of occupancy variability from a pool of similar buildings and make inference for the building of interest (we thereafter summarize this feature as generalization)

- Consideration of the partial correlation between the variability of occupant presence and actions

The natural next step is a search within related literature, looking for an appropriate occupancy representation with these features.

\subsection{Literature review on occupancy modeling}

Although building energy simulation tools continue to evolve and mature, the approach to representing the complex relationships between buildings and occupants has received relatively little attention, and has remained rather rudimentary. Some initiatives came up with more specific diversity profiles ${ }^{2}$ that remain invariable from day to day or week to week (Abushakra \& Claridge, 2008; Bronson, Hinchey, Haberl, \& O'Neal, 1992; Dunn \& Knight, 2005; Roberson et al., 2004). Only fairly recently was occupancy recognized as a major obstacle to producing better predictions (Mahdavi, 2010) and an important contributor to the so-called "performance gap" (Menezes, Cripps, Bouchlaghem, \& Buswell, 2012). The resulting momentum has, for instance, led to a growing body of work that is studying occupancy as stochastic processes, including the IEA-EBC Annex 66 initiative (IEA-EBC Annex 66, 2015), and resulted in tools for generating stochastic demand profiles for occupant services in domestic buildings (Rysanek \& Choudhary, 2015). Popular statistical methods used in this type of research are logistic regression analysis, Markov chain models, Poisson process, and survival analysis (Parys, Saelens, \& Hens, 2011). The following is a brief summary of related work, organized into four major categories: activity based methods, event based methods, Markov chain models and agent-based methods.

Activity based methods rely on activity surveys or time-use data to generate occupancy variables with techniques such as hierarchical clustering to identify typical occupancy patterns (Aerts, Minnen, Glorieux, Wouters, \& Descamps, 2014; Widen et al., 2009). Time-use data, typically obtained from daily activity logs, describe in detail the everyday life of household members as high-resolved activity sequences, such as "awake-sleepingaway" or "appliance-lighting-cooking-dishwashing-drying-ironing-TV", with the former for presence and the latter for domestic occupant services. Potential drawbacks of this approach might be associated with the accuracy of self-reported activity data, the generalization of these activities specific to sampled occupants to a different population, and the invariability of estimated occupancy.

The second category is event based, for instance, the widely referenced Lightswitch model (Reinhart, 2004), specifies that if an occupant arrives and the lighting is off, there might be a switch-on event subject to a predefined probability distribution, but it does not allow for variability between events. Other examples include Lindelof and Morel (2006), who measured the intermediate light switch probability by users as a function of current illuminance levels, and Tabak (2008), who introduced the S-curve method for intermediate activities such as a break or a smoke and defines their probability of occurrence based on the duration of time since the last same event. However, to adopt this method in other organizations, the list of intermediate activities and the behavior patterns of occupants need to be specialized first. Another line of literature within this category has to

\footnotetext{
${ }^{2}$ The name "diversity profile" is used to indicate the industry norm, but the word "diversity" may be misleading: instead of showing diversity of building usage from day to day, standard diversity profiles are static, and assume identical profiles for all weekdays and (separate profiles for) weekends.
} 
do with the window opening behavior of occupants. The window opening probability is usually linked to other environmental variables such as the outdoor temperature (Haldi \& Robinson, 2009; Li, Li, Fan, \& Jia, 2015). Markov chain models have gained popularity in recent occupancy literature. For instance, Page, Robinson, Morel, and Scartezzini (2008) proposed a "generalized" stochastic model for the simulation of occupant presence, followed by various research (Andersen, Iversen, Madsen, \& Rode, 2014; Liao, Lin, \& Barooah, 2012; Tahmasebi \& Mahdavi, 2015; C. Wang, Yan, \& Jiang, 2011). In the original paper of Page et al. (2008), the probabilities of transition from occupied to unoccupied and vice versa in the Markov chain model, are dependent on two user inputs: the profile of the probability of presence and a newly introduced parameter of mobility, which expresses the average ratio of probability of change of presence over the probability of no change. Since these Markov chain models generally originate and have been calibrated from single person offices, they need to be adapted to the more general context of office buildings, in which the most appropriate interpretation of the diversity profile from user input is the aggregated group presence of occupants in a given building thermal zone, which typically consists of several physical spaces. In other words, the profile of the probability of presence is generally not readily available for a single occupant and it is clearly a fallacy to confuse the mean presence of a group of occupants (more predictable) with the probability of presence for each single one of them (less predictable). Another difficulty of occupant presence characterization is the estimation of the parameter of mobility, which is time varying and very specific to the studied occupant. Therefore, generalizing the Markov chain approach requires the establishment of a pool of representative occupants, each with a distinct parameter of mobility, such that any future building can be populated by sampling from a pool of "typical" occupants. The composition of the pool of occupants obviously depends on the building type and tenant organization.

The last category is based on agent-based modeling. Typically, behaviors related to energy consumption are defined, and then factors that contribute to "behavior changes" and their impact are studied (Azar \& Menassa, 2012; Y. S. Lee \& Malkawi, 2014). However, there is a general consensus regarding the difficulty of establishing rules to govern agents' behavior in terms of how they interact with each other and system stimuli.

Most of previously mentioned studies rely on gathering and analysis of occupant presence data directly collected in existing buildings. Typical monitoring methods include ultrasonic and passive infrared detection, direct contact detection, $\mathrm{CO}_{2}$ sensors, camera-based technology, and wireless network based systems (Cali, Matthes, Huchtemann, Streblow, \& Muller, 2015; Rosenblum, 2012). As the granularity of occupancy data increases, major concerns can be raised regarding occupant privacy because of direct occupant tracking methods such as by Shih (2014). Therefore, new data collection methods that build on recent developments in machine learning have been specifically developed for this. "Indirect" occupant positioning methods, such as the one proposed by Zhao, Lasternas, Lam, Yun, and Loftness (2014), are able to accurately predict occupant presence from the data mining of their personal appliance usage without intruding too much into occupants' privacy. However, such an approach also has the generalization issue since the fitted classification model may not apply to a future unique building-tenant combination, especially without deep measurements of the required feature data (e.g. personal usage of computers and lights by a specific occupant) for the fitted classification model.

\subsection{Relevance of stochastic occupancy models}

The imminent question to be answered then is whether occupancy modeling adds significantly to an accurate prediction of building performance. Various researches have reported the effect of occupancy modeling on building energy outcomes (Bonte, Thellier, \& Lartigue, 2014; Duarte, Budwig, \& Van Den Wymelenberg, 2015; 
Saelens, Parys, \& Baetens, 2011). In particular, recent work by Tahmasebi and Mahdavi (2015) evaluates the impact of different occupancy representation on building performance indicators. However, few among these have explicitly made the important distinction between the impact of occupancy variables on direct and indirect energy outcomes. It is an increasing trend in commercial buildings that control is left to automated systems, leaving little to human intervention, except for direct electricity use for lighting (in some cases) and appliances (in most cases). Another issue to consider is the level of interaction between the operation of building systems and the spatial and temporal variability of occupancy. If controllability of the HVAC system is distributed and nimble, one can expect a strong dependency between the building operation and occupancy (Goyal, Barooah, \& Middelkoop, 2015; Oldewurtel, Sturzenegger, \& Morani, 2013). However, the current market is still dominated by conventional HVAC systems with a central and fixed control and limited response to zonal and temporal variability. Adjusting outdoor air supply, controlling zonal temperature or shutting down unused zones based on occupancy, or controlling operable windows on a zone-by-zone basis is typically not possible. A direct response of the building operation to occupancy is mostly absent or at best "muted" in conventional buildings. Therefore, we argue that in most current buildings, the occupants' impact on HVAC system consumption is only the indirect effect of their presence and control of lighting and appliances. Another important consideration is that besides the imperfect knowledge of occupancy, many other uncertainties persist in building energy models. They stem from two major sources. The first is uncertainty related to the physical properties of the building components and system parameters, as reported by many authors (de Wit \& Augenbroe, 2002; Heo, Choudhary, \& Augenbroe, 2012; Hopfe \& Hensen, 2011; Macdonald \& Strachan, 2001; L. P. Wang, Mathew, \& Pang, 2012). Another source of uncertainty is the discrepancy of the model itself. State-of-the-art energy models represent complex physical processes through certain levels of abstraction and simplification. We categorize this type of uncertainty as "model form uncertainty", which has been shown to be an important contributor to the socalled "performance gap" (Sun, 2014). As a consequence, the impact of occupancy models cannot be examined without acknowledging the role of all other sources of uncertainty. Rather, the sensitivity of occupancy models should be ranked against other uncertainties.

In summary, in this paper, we address two research gaps that seem to emerge out of the breadth of previous efforts.

(1) The building energy simulation community lacks guidance for an appropriate representation of occupancy, taking into account the modeler's level of knowledge. A generalizable framework that enables inference for estimating the occupancy of future unrealized buildings with knowledge from current building stocks is yet to be developed.

(2) The relevance of occupancy models on various energy outcomes of interest under uncertainty, with a clear distinction between direct and indirect energy consumption has yet to be studied.

In this paper, we present a solution to both issues, with emphasis on the two major occupancy variables: (1) occupant presence and (2) combined lighting and appliance electricity consumption. Other occupancy and scenario variables in general (if needed) may be added and treated in the same way.

\section{PROPOSED MODELING FRAMEWORK}

Strategies for defining occupancy variables for modelers with totally ignorant information or perfect information are expected to be straightforward. Therefore, we concentrate in this paper on guiding modelers with intermediate knowledge through the creation of an adequate representation of building occupancy. Recall that we 
have implicitly distinguished between a potentially biased and uncertain view of the modeler on the typical building occupancy, and a general lack of knowledge about the day-to-day fluctuations of occupancy. Such distinction motivates us to model the mean profile and variability of occupancy variables separately with different models.

\subsection{Modeling of mean occupancy profiles}

The major assumption is that we do not second-guess an energy modeler with intermediate knowledge about typical building occupancy. In other words, we assume the standard diversity profile has been carefully consolidated with the building owner and is consistent with on-site audits or tenant survey results. This is not to say that these inputs are not subject to inaccuracy. On the contrary, the goal of the model is to generate stochastic samples of mean occupancy profiles based on the modeler's belief about the uncertainty associated with the estimated diversity profiles.

The model of mean occupancy profiles that we propose assumes that the 24-hour profiles follow a multi-variant Gaussian distribution $N(\mu, \Sigma)$ with the value at each hour being a random variable. Since such a distribution is fully specified by the mean vector $\mu$ and covariance matrix $\Sigma$, we specify $\mu$ with the standard input of diversity profiles and diagonal elements of $\Sigma$ with the variance estimates by the modeler. It is still problematic to generate multi-variant samples since elements of the matrix $\Sigma$ other than diagonals remain unspecified. These entries determine the correlation between hourly values and they combine to drive the smoothness of the resulting mean occupancy profiles. Our approach to specifying the correlation is for obvious reasons data-driven. We analyze data from ASHRAE Research Project 1093-RP (Abushakra, Sreshthaputra, Haberl, \& Claridge, 2001) to establish a correlation matrix $\mathrm{C}$ between each hourly random variable and derive the covariance matrix with the following equation:

$$
\Sigma=\mathrm{D} * \mathrm{C} * \mathrm{D},
$$

where matrix $\mathrm{D}$ is a diagonal matrix comprised of the estimate standard deviation of the profile value of each hour. Then a multi-variant Gaussian sample is the following:

$$
x=\mu+L u,
$$

where $L$ is the Cholesky decomposition of the positive definite symmetric covariance matrix $\Sigma=L L^{T}$, and $u$ is a vector composed of multiple independent Gaussian random numbers, each following the standard normal distribution $N(0,1)$. The 24-dimensional sample $x$ represents the mean occupancy of the building of interest.

The above derivation relies on the modeler's estimate of the uncertainty of the standard diversity profiles. Informative estimates can be based on pure judgement, a mixture of data and judgement, or data alone. We provide guidance to modelers with concepts borrowed from Bayesian literature (Lunn, Jackson, Best, Thomas, \& Spiegelhalter, 2012). It is well known that in a Bayesian approach, prior information is updated with information from acquired data. In some special cases, the posterior mean is a weighted average of the prior mean and observed data, with the weights determined by the relative contribution of the prior. The prior information could be interpreted as representing "implicit data", in other words, the more concrete prior information infers larger "effective prior sample size". Such is the case in our application: the more reliable the modeler's information, the less bias and uncertainty with the diversity profile should be expected, that is, higher confidence in the estimates of building occupancy is in some way equivalent to possessing more "implicit data". However, for a non-existent and unique building, the modeler has to rely on his subjective judgement for an expression of uncertainty. The elicitation of subjective uncertainty distribution can be intimidating and is no easy task. We refer the modelers to 
O'Hagan et al. (2006) for further reference, as this is not the main focus of this paper. The "mixture of data and judgement" approach will be accounted for in a Bayesian framework that incorporates a tradeoff between subjective beliefs (effective prior samples) and collected actual data samples. Nevertheless, in order to preserve objectivity of this paper, we refrain from expressing our subjective beliefs, but take a data-driven approach by applying a resampling technique called "bootstrapping" to mimic the modeler's estimate of the standard deviation of the hourly profile values in matrix D. The basic idea of bootstrapping is that the accuracy of summary statistics about a population from sample data (from sample to population) can be inferred by resampling the sample data with replacement (from resample to sample). The simplest bootstrap method starts by computing a resample with replacement of the same size as the original data set. This process is repeated a large number of times, and for each of these bootstrap resamples we calculate a bootstrap mean. We now have a large population of bootstrap means, which leads to an estimate of how much the mean varies. Success of bootstrapping rests on the premise that inference of the empirical distribution of $\hat{\jmath}$, given the resampled data, resembles closely that of the true probability distribution $J$, given the original data. Note that it is bias in the original sample data that introduces bias to the bootstrapping inference, while it is variability in the original sample data that leads to the uncertainty of the mean. We therefore propose two scenarios to reflect different levels of confidence of the modeler in his estimate. The first scenario is constructed by choosing two arbitrary days (small effective prior sample size) from monitored occupancy variables as the original sample data and applying the bootstrapping technique to them. It is expected that in this low-confidence case, there will be large bias and variance associated with the mean. The second high-confidence scenario is constructed with a much larger sample size of fifteen days (large effective prior sample size), which will lead to low bias and uncertainty with the bootstrap estimate of the mean.

\subsection{Meta-analysis of occupancy variability}

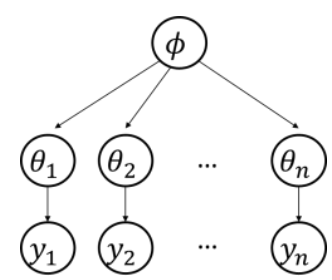

Figure 2 A basic hierarchical model

Recall that we assume that a modeler with intermediate knowledge is not aware of the day-to-day variability of occupancy variables, so we need to borrow knowledge from previously thoroughly studied buildings and apply to the one of interest. This can only be accounted for in a general hierarchical data inference model depicted in Figure 2. Suppose data $y_{i}$ are a group of observations from building $i$, which we assume to be dependent on the corresponding building parameter $\theta_{i}$ (specific value for each building). We learn about $\theta_{i}$ not only through direct information $y_{i}$, but also through indirect information, which comes from the remaining $y_{j}: j \neq i$, via a higherlevel distribution, which applies to the whole population of studied buildings parameterized by $\phi$. We therefore propose a meta-analysis that builds upon data from previous research studies, from which we extract and learn the characteristics of occupancy variability and quantify the degree of similarity between them, eventually enabling the inference of building-specific parameters for the future building of interest. 


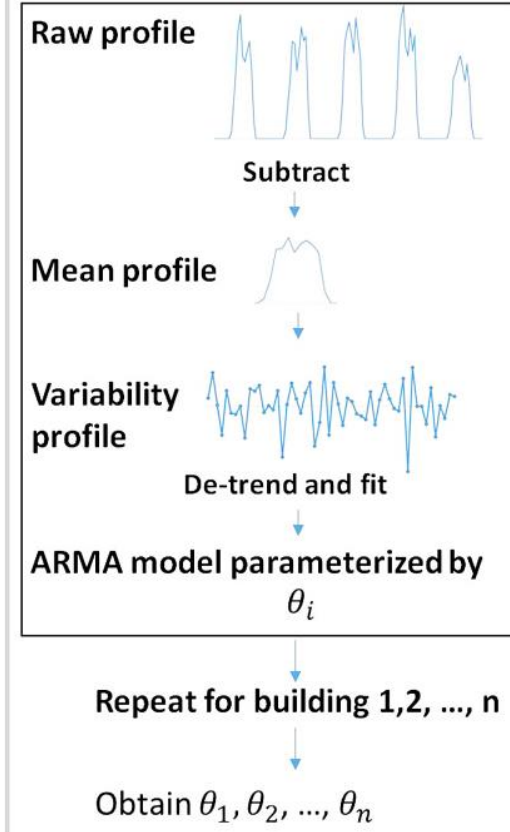

Non-informative prior for population parameter $\phi$

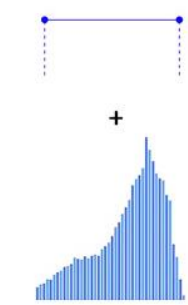

Data $\theta_{1}, \theta_{2}, \ldots, \theta_{n}$

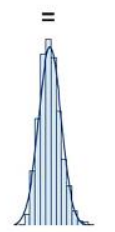

Posterior estimation for $\phi$

Stage 2: Knowledge Extraction
Sample a $\phi_{j}$ from its distribution, and then sample a $\theta_{j}$ from the population distribution indexed by $\phi_{j}$

Simulate variability profile with the ARMA model determined by $\boldsymbol{\theta}_{j}$

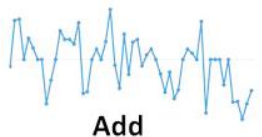

Mean profile

Realized profile

Repeat for sample $1,2, \ldots, \mathrm{N}$

Figure 3 Proposed framework for a meta-analysis

We organize the framework into three stages: an analysis stage, a knowledge extraction stage, and an inference stage, as depicted in Figure 3. Given collected occupancy data about the number of occupants and lighting/appliance consumption per time step for a total of $\mathrm{N}$ weekdays, in the analysis stage, we first normalize these data by the maximum number of occupants and the maximum lighting/appliance consumption from design information $^{3}$, which leads to hourly profiles $O_{t}$ and $L_{t}$ normalized between zero and one. Now that we have N samples for each hour of a day for both variables, we derive average occupancy variable values for each hour, which compose a typical weekday. After populating two time series $\overline{O_{t}}$ and $\overline{L_{t}}$ with each day being identical, i.e. the constructed typical weekday, we subtract $\overline{O_{t}}$ and $\overline{L_{t}}$ from the original series $O_{t}$ and $L_{t}$ and obtain the residual series for presence $X_{t}$ and lighting/appliance usage $Y_{t}$, which account for day-to-day occupancy variability. $X_{t}$ and $Y_{t}$ are analyzed with linear transfer function models such that building-specific parameters $\theta_{i}$ are derived. In the knowledge extraction stage, building-specific parameters from multiple buildings are analyzed to estimate the population distribution, parameterized by $\phi$. In the final inference stage, for each probable realization of the future building of interest, we perform hierarchical sampling by first sampling a $\phi_{j}$ from its posterior distribution, and then sampling a $\theta_{j}$ from the population distribution indexed by $\phi_{j}$. With the building-specific parameter $\theta_{j}$, we can reproduce occupancy variability with time series models. Having established the general framework, we now move on to more specifics on linear transfer function models.

Most physical processes exhibit inertia and do not change that quickly. This, combined with the sampling frequency, often makes consecutive observations correlated. Such serial dependence between consecutive observations, which is common in occupancy data, is called autocorrelation. Autocorrelation is beyond the assumption of independence for most statistical methods, but can be easily taken into account with time series

\footnotetext{
${ }^{3}$ A reasonable estimate of the maximum number of occupants and the maximum lighting/appliance consumption can, for instance, be based on the number of seats and lighting/appliance fixtures from the design documentation. These numbers can be arbitrarily chosen as long as the modeler realizes, for instance, that the predicted maximum profile value of one corresponds to the maximum number of occupants in the space.
} 
methods. Therefore, the application of time series methods to the study of occupancy seems to be natural. For instance, Schweigler et al. (2009) was able to create a seasonal ARIMA model and robustly forecast a clinic's emergency department (ED) bed utilization 4 and 12 hours in advance for three different EDs. Inspecting the limited research efforts that are available on the topic of occupancy modeling with time series models, we are able to identify the following two obstacles. First, occupancy in general is non-stationary and highly dependent on time of day, so standard ARIMA models cannot be directly applied without data pre-processing, which may be the reason why literature on this topic is scarce. This obstacle is overcome since we concentrate on the modeling of occupancy variability, which is generally stationary and independent of time. The second obstacle is that of the times series models developed around this topic, few allow for the interplay between occupant presence and their operation of lighting and appliances, which has to be examined together with a multidimensional time series technique such as a linear transfer function model detailed below.

Consider two time series $\left(X_{t}, Y_{t}\right) . X_{t}$ is the input time series and $Y_{t}$ is the output time series. A linear transfer function model has the following form:

$$
Y_{t}=v(B) X_{t}+N_{t}
$$

where $B$ is the backshift operator, $v(B)=v_{0}+v_{1} B+v_{2} B^{2}+\cdots$, and $N_{t}$ is an $\operatorname{ARIMA}(p, d, q)$ process. The operator $v(B)$ is called the transfer function of the model with weights $v_{0}, v_{1}, v_{2}, \ldots$ The system is stable if $\sum_{i=1}^{\infty}\left|v_{i}\right|<\infty$. In the form of difference equations, Equation 3 is written as

$$
Y_{t}=\delta_{r}(B)^{-1} \omega_{s}(B) B^{b} X_{t}+N_{t}
$$

where $\delta_{r}(B)=1-\delta_{1} B-\delta_{2} B^{2}-\cdots-\delta_{r} B^{r}, \omega_{s}(B)=\omega_{0}-\omega_{1} B-\omega_{2} B^{2} \ldots-\omega_{s} B^{s}$ and $b$ is the delay of $b$ time lags.

Suppose for the moment that $Y_{t}=v(B) a_{t}+N_{t}$, i.e. $X_{t}=a_{t}$, which is white noise independent of $N_{t}$. Then it is easy to show that the transfer function is proportional to the cross correlation function of the two series. Now suppose that $X_{t}$ is an $A R(p)$ process, that is $\phi(B) X_{t}=a_{t}$, where $\phi(B)$ is a polynomial. Multiplying both sides of Equation 3 by $\phi(B)$, we obtain $\phi(B) Y_{t}=v(B) a_{t}+\phi(B) N_{t}$. That is for the process $Y_{t}^{\prime}=\phi(B) Y_{t}$, by computing the cross correlation between $Y_{t}^{\prime}$ and $a_{t}=\phi(B) X_{t}$, we can estimate the transfer function. This is called the pre-whitening of the input. As such, we can summarize the procedures of estimating a linear transfer function model from data:

(1) Fit an ARMA model to the input series $X_{t}$, retain model coefficients for use in Step 2 and the fitted residuals $\widehat{a_{t}}$ for use in Step 3.

(2) Apply the ARMA operator determined in Step 1 to determine the pre-whitened output series $Y_{t}^{\prime}$.

(3) Use the cross correlation function between $\widehat{a}_{t}$ and $Y_{t}^{\prime}$ in Step 1 and 2 to suggest an appropriate form for the component of the transfer function $v(B)=\delta_{r}(B)^{-1} \omega_{s}(B) B^{b}$, especially the estimated time delay $b$.

(4) Obtain model coefficients by fitting a linear regression of the following form:

$$
Y_{t}=\sum_{k=1}^{r} \delta_{k} Y_{t-k}+\sum_{k=1}^{s} \omega_{k} X_{t-k-b}+u_{t},
$$

where $u_{t}=\delta_{r}(B) N_{t}$.

Retain the residuals $\widehat{u_{t}}$ for use in Step 5.

(5) Applying the moving average transformation $\delta_{r}(B)^{-1}$ to the residuals $\widehat{u_{t}}$ to find the noise series $N_{t}$, and fit an $A R M A$ model to it. 


\section{SENSITIVITY ANALYSIS CASE STUDY}

Following the proposed framework, we are ready to conduct a sensitivity analysis case study. Specifications of the case study will be introduced in the order of data collection (Section 3.1), case study building description (Section 3.2), variants of occupancy representation (Section 3.3), other sources of uncertainty (Section 3.4), and sensitivity analysis technique (Section 3.5).

\subsection{Data collection}

Despite the effective framework that we propose above, we realize that the research community possesses only sporadic data sources of building occupancy, especially those that contain detailed interval data regarding both occupant presence and their control of lighting and use of appliances. Therefore, we rely on our own dataset and for now only analyze the characteristics of one building as an illustration of the analysis stage (Stage 1 in Figure 3 ) in the framework. The measurements are conducted from January $11^{\text {th }}$ to February $7^{\text {th }}$ in a medium-size mixed use building on the Georgia Tech campus. The building has an area of about 45000 square foot divided into office and lab spaces. At each entrance of the building, occupancy sensors (in Figure 4 below the "exit" sign) based on infrared sensing technology are installed. These directional sensors detect the passage of people, determine their directions, and then send signals to minicomputers (in Figure 4 above the "exit" sign), where the signals are transformed into counting data and stored until a download command is received. With these sensors, we derive the total number of occupants inside the building by subtracting the accumulated number of exits from that of entrances at each time step. Data are collected with a five-minute time interval. Furthermore, the building is equipped with electricity sub-meters so that we can collect the lighting and appliance consumption data in five-minute time interval.

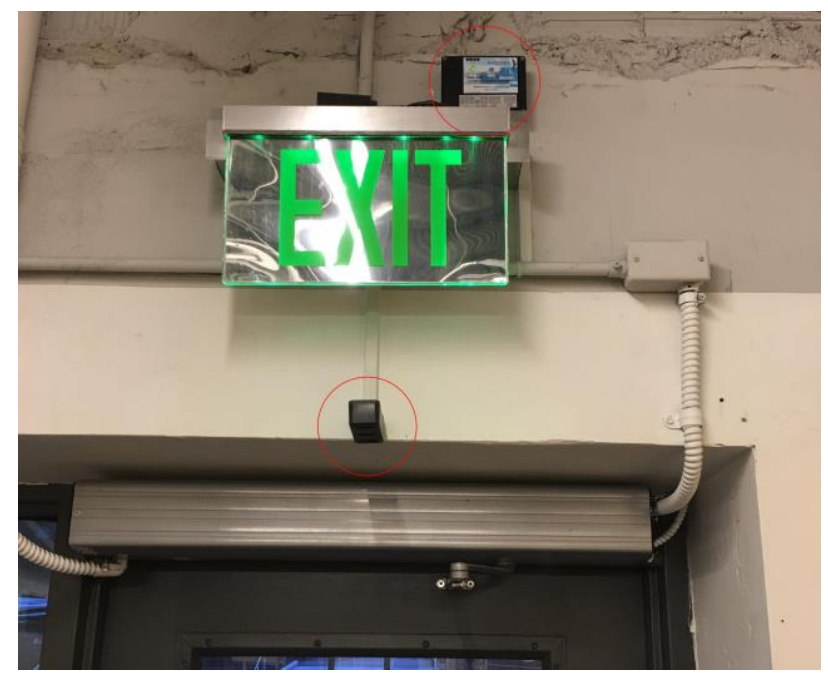

Figure 4 Occupancy sensor connected with a minicomputer above a building entrance

\subsection{Case study building description}

In order to evaluate the role of occupancy variables in the energy use of office buildings, we conduct a sensitivity analysis based on the medium office building from the DOE reference building pool (Deru et al., 2011), as shown in Figure 5. The building has three floors, and each consists of a core zone and four perimeter zones. The system in the building includes a central packaged air conditioning unit with a gas furnace for heating and cooling, and variable air volume (VAV) terminal boxes with reheat for air distribution. In the sensitivity analysis, we study quantities of interest such as aggregated cooling/heating energy in Atlanta, aggregated 
behavior") that are used to compute demand charges by the utility or to verify the benefit of enrolling in a demand response program. The lighting and appliance consumption metric highlights the impact of occupancy on direct energy outcomes, while HVAC system consumption studies the indirect effect. Power duration curves show the percentage of time of the considered period when whole building demand exceeds a certain power level. The whole building demand includes both direct (lighting/appliance) and indirect (HVAC) end uses.

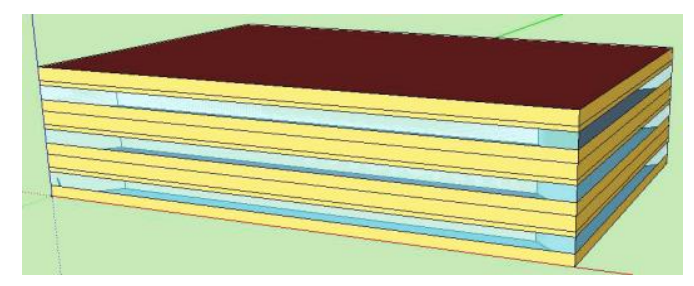

Figure 5 DOE reference medium office building

\subsection{Variants of occupancy representation}

Recalling our definition of information available to a typical modeler about building occupancy in Figure 1, we now specify six levels of occupancy models accordingly. Level 1 corresponds to the "total ignorance" case (Point A). At this point, any plausible assumption is as good as any, so the modeler's best bet is to use the typical weekday and weekend diversity profiles suggested in the ASHRAE guideline (ASHRAE, 2004). Given the lack of better knowledge, there is no justification to assume day-to-day variability, so we suggest using identical profiles for all weekdays and (separate profiles for) weekends throughout the simulation. Level 2 reflects a "midspectrum" case (moving from Point A to B on Figure 1), in which the modeler is still ignorant of occupant presence but has accurate knowledge of the mean lighting/appliance usage of the buildings, represented by usage profiles with each day being the identical typical weekday derived from our dataset. Level 3 moves further to Point $\mathrm{C}$ along the curve in Figure 1, and is linked to a modeler that has accurate knowledge about the mean profiles of both occupancy variables. Level 4 mimics the "perfect knowledge" case, in which we model occupancy as a fully deterministic time series with collected actual data that show day-to-day fluctuations. As such, Level 1 through 4 have dealt with the full spectrum of occupancy information available to a typical modeler. However, note that Level 2 and 3 are somewhat artificial in the sense that the assumed perfect knowledge about the mean profiles of both occupancy variables is generally impractical, but contrasting Level 3 with Level 4 will allow us to inspect the relevance of occupancy variability on energy outcomes, and a comparison between Level 2 and 3 isolates the effect of occupant presence from lighting/appliance usage. In order to represent a modeler's intermediate information more realistically, we introduce two additional levels, which augment Level 2 and 3 in two ways. First, uncertainty about the mean profiles of occupancy variables is introduced with Level 5 representing a low-confidence scenario and Level 6 a high-confidence scenario. As mentioned in Section 2.1, low confidence with the estimates leads to high bias and variance with the mean profiles, and the same principle applies for the high confidence scenario. Second, day-to-day occupancy variability, is overlaid on top of the samples of mean occupancy profiles in both Level 5 and 6 to show day-today fluctuations.

Each of the above levels is associated with identical uncertainty in terms of building and system parameters and model forms, so that we can examine the role of occupancy variables in relation to other confounding factors. It may be necessary to emphasize that occupancy variables at Level 1 through 4 are represented as static time series in each sample simulation run of our uncertainty analysis with Latin hypercube sampling (LHS). However, each sample simulation at Level 5 and 6 chooses a different sample of mean occupancy profiles, and then we add 
the static time series with another times series generated by the transfer function model for occupancy variability. The resulting profile in each sample run at Level 5 and 6 never repeats itself but reproduces the characteristics of the actual occupancy dataset that we collected.

The following table again summarizes and highlights the difference between the six levels.

Table 1 Variants of occupancy representation

\begin{tabular}{|c|c|c|c|}
\hline Levels & Presence and lighting/appliance (L/A) representation & $\begin{array}{l}\text { Variance between } \\
\text { sample simulations }\end{array}$ & $\begin{array}{l}\text { Day-to-day } \\
\text { variability }\end{array}$ \\
\hline \multirow{2}{*}{$\begin{array}{c}\text { Level } \\
1\end{array}$} & Presence: mean profile from ASHRAE standard & \multirow{2}{*}{ N/A } & \multirow{2}{*}{ N/A } \\
\hline & L/A: mean profile from ASHRAE standard & & \\
\hline \multirow{2}{*}{$\begin{array}{c}\text { Level } \\
2\end{array}$} & Presence: mean profile from ASHRAE standard & \multirow{2}{*}{ N/A } & \multirow{2}{*}{ N/A } \\
\hline & L/A: mean profile derived from collected data & & \\
\hline \multirow{2}{*}{$\begin{array}{c}\text { Level } \\
3\end{array}$} & Presence: mean profile derived from collected presence data & \multirow{2}{*}{ N/A } & \multirow{2}{*}{ N/A } \\
\hline & L/A: mean profile derived from collected L/A data & & \\
\hline \multirow{2}{*}{$\begin{array}{c}\text { Level } \\
4\end{array}$} & Presence: collected presence data & \multirow{2}{*}{ N/A } & \multirow{2}{*}{ Applicable } \\
\hline & L/A: collected L/A data & & \\
\hline \multirow{2}{*}{$\begin{array}{l}\text { Level } \\
5\end{array}$} & $\begin{array}{l}\text { Presence: mean profile sampled from the multi-variant Gaussian } \\
\text { distribution (high bias and variance), variability generated (together } \\
\text { with L/A) with the transfer function model }\end{array}$ & \multirow{2}{*}{ Applicable } & \multirow{2}{*}{ Applicable } \\
\hline & $\begin{array}{l}\text { L/A: mean profile sampled from the multi-variant Gaussian distribution } \\
\text { (high bias and variance), variability generated (together with presence) } \\
\text { with the transfer function model }\end{array}$ & & \\
\hline \multirow{2}{*}{$\begin{array}{c}\text { Level } \\
6\end{array}$} & $\begin{array}{l}\text { Presence: mean profile sampled from the multi-variant Gaussian } \\
\text { distribution (low bias and variance), variability generated (together } \\
\text { with L/A) with the transfer function model }\end{array}$ & \multirow{2}{*}{ Applicable } & \multirow{2}{*}{ Applicable } \\
\hline & $\begin{array}{l}\text { L/A: mean profile sampled from the multi-variant Gaussian distribution } \\
\text { (low bias and variance), variability generated (together with presence) } \\
\text { with the transfer function model }\end{array}$ & & \\
\hline
\end{tabular}

\subsection{Other sources of uncertainty}

Until now, our approach has focused on modeling temporal variability, as this is the main thrust of the paper. Indeed, the presence data driving the analysis is assumed available at a whole building level, thus reflecting the temporal variability of occupant presence in the whole building. Total occupancy is proportionally allocated to the building zones. In reality, this proportionality assumption rarely holds, and as a result it is relevant to inspect the effect of spatial variability, i.e. knowing how many people are in the building, but not knowing where they are exactly. We introduce an arbitrary uncertainty factor $d$ to represent the spatial variability of occupant presence over the three core zones in the office building, such that in each simulation run, the total number of occupants $(3 P)$ remains the same with $d \mathrm{P}$ occupants in Zone 1 and $\frac{(3-d) P}{2}$ occupants in Zones 2 and 3 respectively. Since we don't have enough information to justify a factor $d$ that varies from day to day, we assume it is static in each simulation run, but this implementation still ensures that factor $d$ (uniformly distributed in the interval from 0.7 to 1.3 ) creates significant spatial variability of occupant presence. 
Another factor that is often viewed as part of the set of occupancy variables is variable thermostat settings, i.e. expressing occupant control of indoor temperatures. We have not focused on this variable because of lack of monitored data, but it goes without saying that were data available, it could be treated in our framework in the same manner as explained above. In the scope of this paper, it is relevant to compare the relative impact of the variability of thermostat settings versus occupancy variability. As occupants have only limited controllability over thermostats, that is, occupants typically can only overwrite the centrally controlled value for a short period of time within a $1 \sim 2^{\circ} \mathrm{C}$ band and over time buildings are effectively centrally controlled, we are interested to show the relative impact of temperature settings within this narrow band. As we are only interested in the first order effect, the implementation is simply that the heating or cooling set-point is determined by a triangular distribution of plus or minus $1^{\circ} \mathrm{C}$ around the original value and stays constant throughout the year.

\subsection{Sensitivity analysis technique}

The concept of "sensitivity analysis" is quite broad. For a review of typical sensitivity analysis methods used in the domain of building performance simulation, readers are referred to the review paper by Tian (2013). In this paper, we apply a variance-based sensitivity analysis methodology, where a first-order or main-effect sensitivity index represents the expected reduction in the percentage of output variance, if the parameter under investigation could be known or fixed. Sensitivity indexes of uncertain parameters are computed with the techniques of Sun, $\mathrm{Gu}, \mathrm{Wu}$, and Augenbroe (2014). The sensitivity analysis technique is premised on Latin hypercube samples from the previous uncertainty analysis and includes two steps: (1) use lasso (Tibshirani, 1996) as a screening procedure to remove insignificant parameters, (2) compute global sensitivity indexes in percentage with a variance-based method. The index of an uncertain parameter under investigation will be interpreted as the percentage of variance of the output explained by this parameter.

\section{RESULTS AND DISCUSSION}

\subsection{Profiles for occupancy variables}

We collected and analyzed measurement data for four consecutive weeks from January $11^{\text {th }}$ to February $7^{\text {th }}, 2015$ without the interruption of school holidays. We excluded weekends from this study since the variability of occupancy is quite small during those days. Figure 6 shows the occupancy profiles during twenty weekdays, which happen to show quite large day-to-day fluctuations.
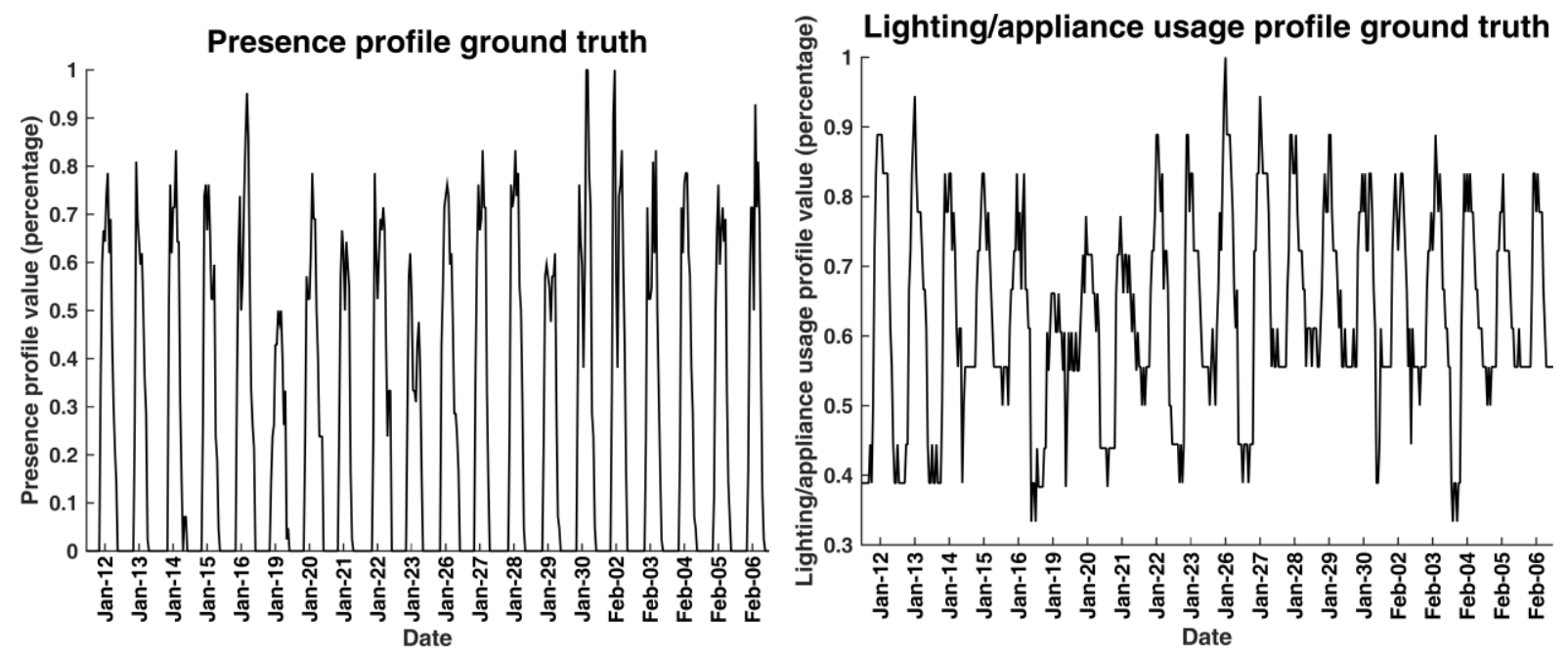


\subsection{Correlation matrix in the model of mean occupancy profiles}

The ASHRAE project (Abushakra et al., 2001) reports weekday mean diversity profiles at the building level.

Figure 7 depicts the range of lighting and appliance usage profiles in a total of 15 buildings. The hourly correlation is derived from this dataset to inform the generation of the covariance matrix for lighting and appliance usage. Since the dataset does not have extensive presence information, the same correlation matrix is also used for generating mean presence profiles out of simplicity. The correlation matrix values are illustrated in Figure 8. As we can see, the correlation of hourly value is highest around early morning, noon and night.

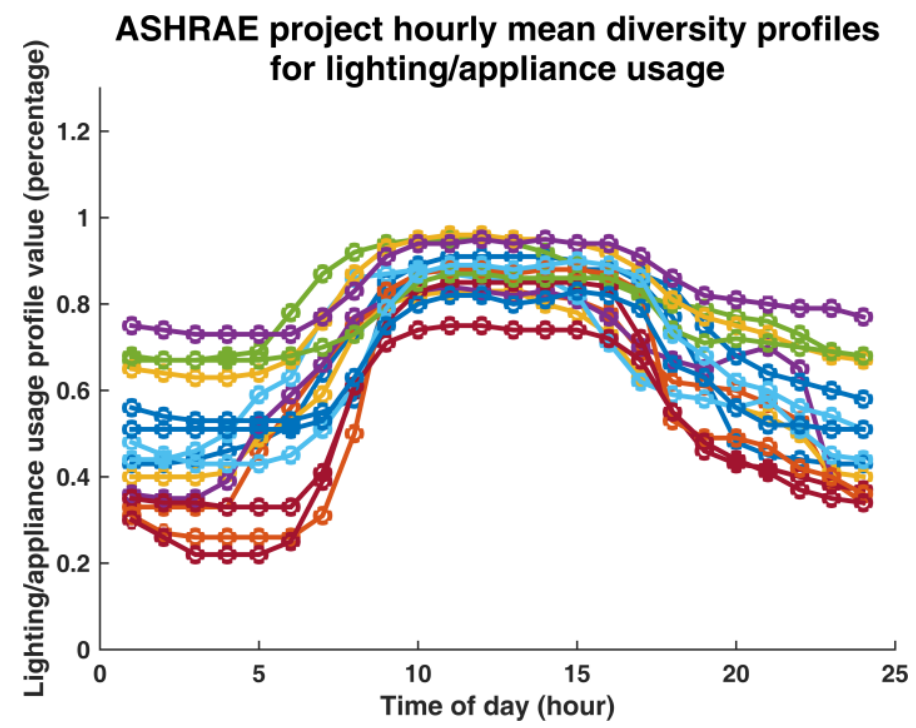

Figure 7 Hourly mean diversity profiles for lighting/appliance usage in the ASHRAE project

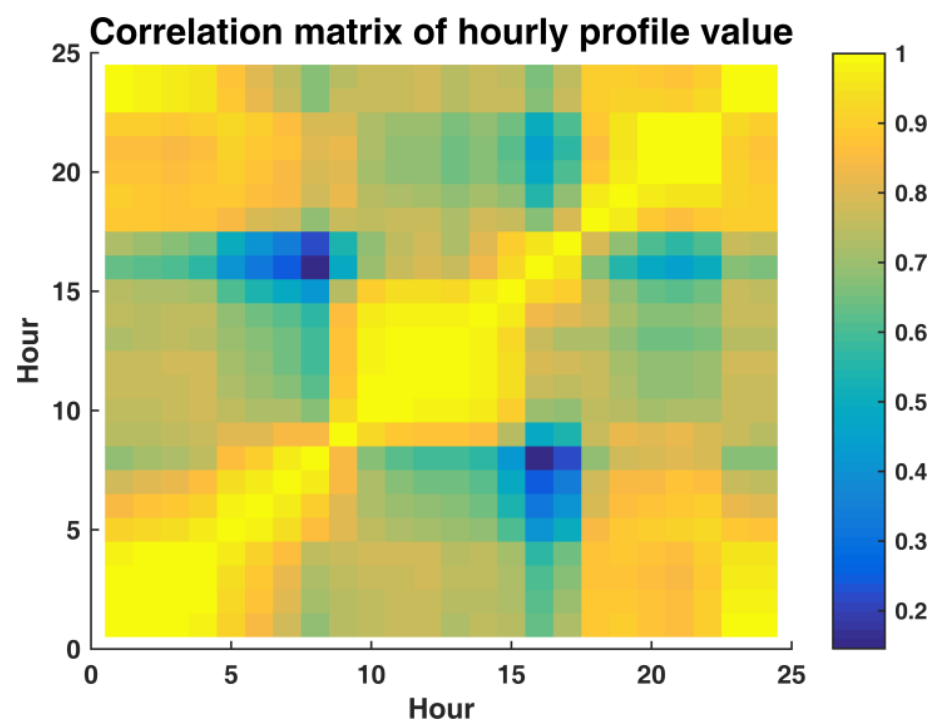

Figure 8 Correlation matrix in the model of mean occupancy profiles

\subsection{Estimation of the linear transfer function model}

Recall that we use linear transfer function models to analyze the variability of occupancy variables, so the profiles in Figure 6 have to be further pre-processed. We first subtract the mean occupancy series, remove the non-occupied hours and then further de-trend the series with a linear regression with the week number as a

\footnotetext{
${ }^{4}$ The term "ground truth" is often used in machine learning literature, to denote information directly observed rather than predicted.
} 
categorical independent variable. Table 2 summarizes the statistics for the two regressions. By observing the $\mathrm{p}$ value, we identify that in Week 2 , both presence and lighting/appliance usage are lower than other weeks. In addition, lighting/appliance usage in Week 3 is higher than other weeks. After this procedure, the residuals of the two series, i.e. $X_{t}$ and $Y_{t}$ are stationary, as shown in Figure 9.

Table 2 Statistics of regression models

\begin{tabular}{|c|c|c|c|}
\hline Series & Predictor & Coefficients & P Value \\
\hline Presence & Intercept & 0.014 & 0.39 \\
\cline { 2 - 4 } & Week 2 & -0.067 & 0.003 \\
\cline { 2 - 4 } & Week 3 & 0.01 & 0.63 \\
\cline { 2 - 4 } & Week 4 & 0.0004 & 0.99 \\
\hline Usage & Intercept & -0.0017 & 0.83 \\
\cline { 2 - 4 } & Week 2 & -0.0035 & 0.002 \\
\cline { 2 - 4 } & Week 3 & 0.038 & 0.001 \\
\cline { 2 - 4 } & Week 4 & 0.004 & 0.70 \\
\hline
\end{tabular}
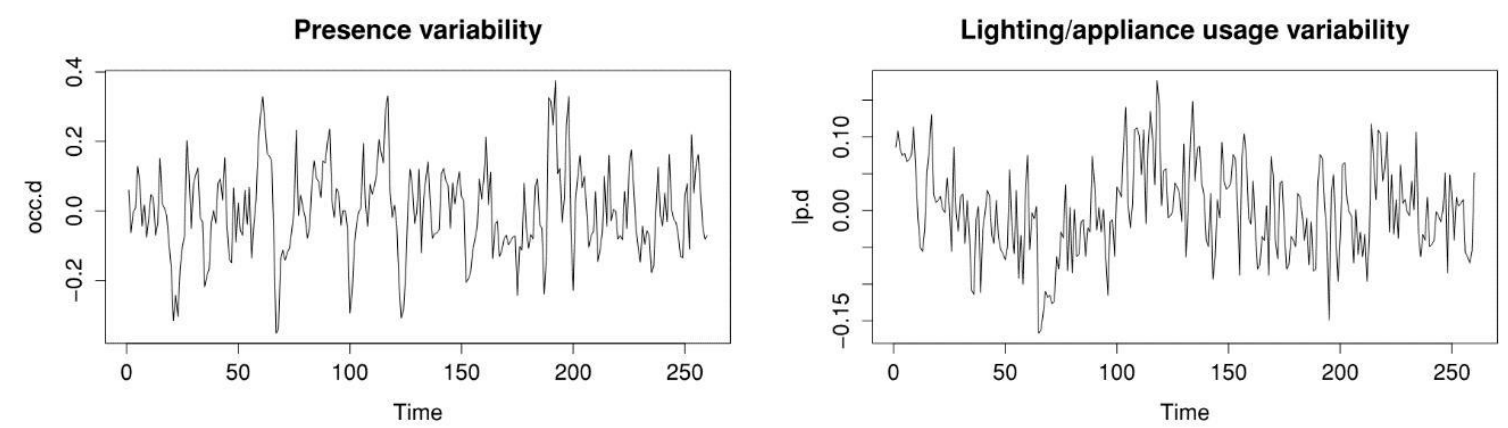

Figure 9 De-trended presence variability (left) and lighting/appliance usage variability (right)

Standardized Residuals
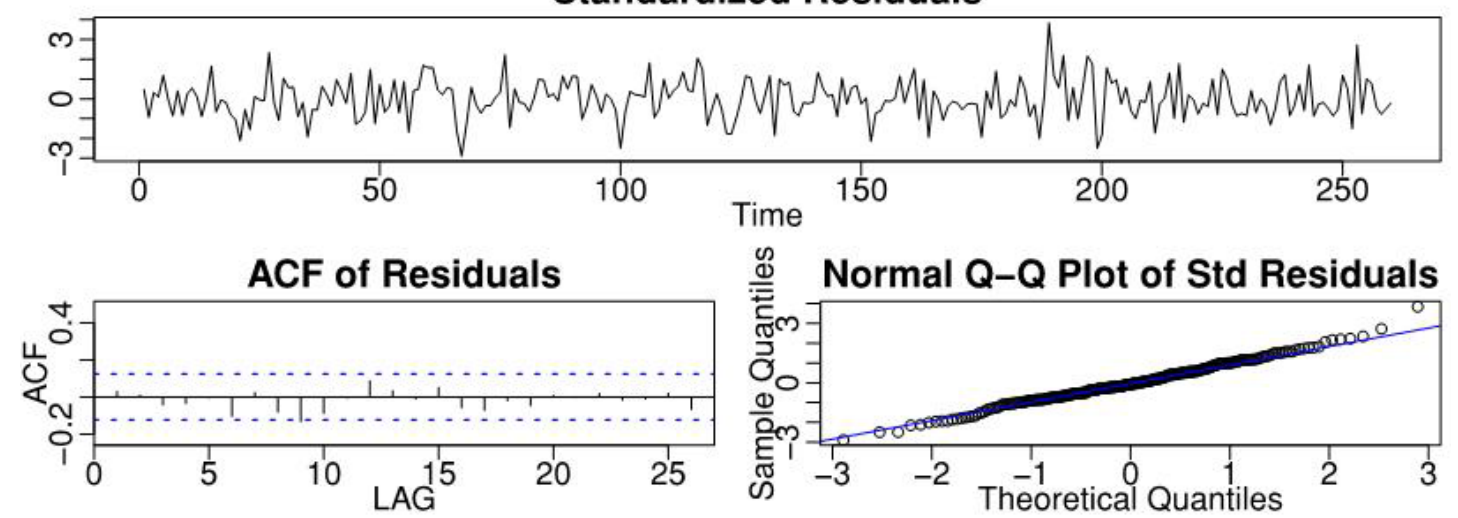

$p$ values for Ljung-Box statistic

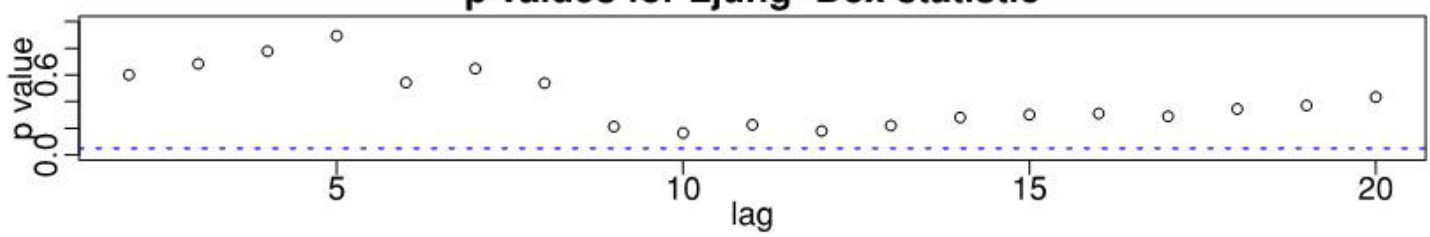

Figure 10 Diagnostic plots for the AR(1) model for $X_{t}$ 
Following the procedures for estimating a linear transfer function, we choose an $A R(1)$ model after model selection for the presence variability series $X_{t}: X_{t}=0.59 X_{t-1}+a_{t}$, where $\widehat{\sigma_{a_{t}}^{2}}=0.01082$. Diagnostic plots in Figure 10 show that the standardized residuals seem normally distributed, and that auto-correlations of the residuals $\widehat{a_{t}}$ seem to have been removed, since the Ljung-Box test cannot reject the null hypothesis that the model is adequate at a 0.05 level. Applying the operator $\phi(B)=(1-0.59 B)$ to the output series $Y_{t}$, we get the pre-whitened series $Y_{t}^{\prime}$. Figure 11 depicts the cross-correlation between $Y_{t}^{\prime}$ and $\widehat{a_{t}}$, with an apparent shift of $b=1$ hour. Through trial and error, it seems plausible to hypothesize a model of the form $v(B)=(1-$ $\left.\delta_{1} B\right)^{-1} B^{1}$, and the corresponding regression estimate is

$$
Y_{t}=0.46 Y_{t-1}+0.09 X_{t-1}+u_{t},
$$

where $\widehat{\sigma_{u_{t}}^{2}}=0.0029$. It also turns out $\widehat{u_{t}}$ is basically white noise so that the final Step 5 is not necessary. Note that in this particular case, the variability of lighting/appliance usage is only related to usage and presence in the previous hour, but this may not always be the case.

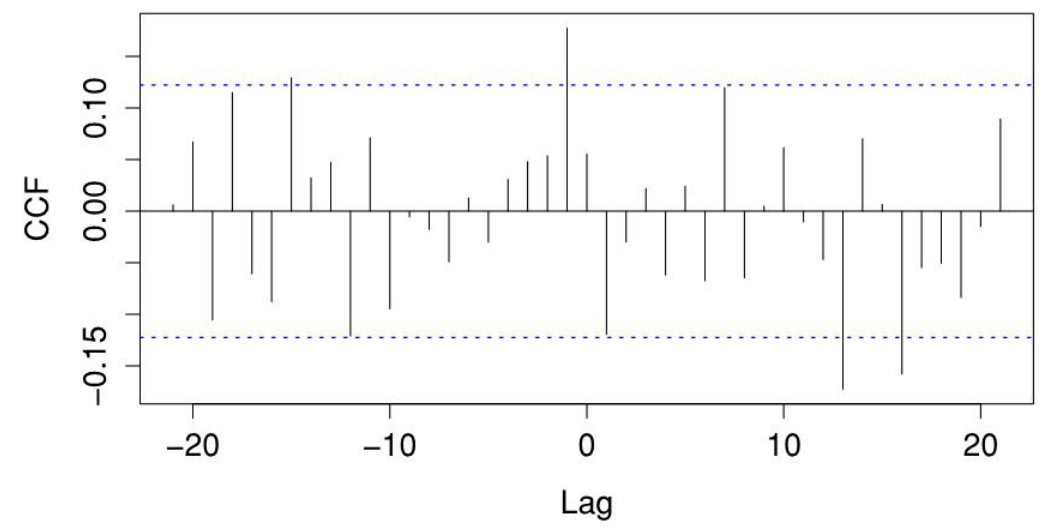

Figure 11 Cross-correlation between $Y_{t}^{\prime}$ and $\widehat{a_{t}}$

Comparing the scale of $\widehat{\sigma_{a_{t}}^{2}}$ and $\widehat{\sigma_{u_{t}}^{2}}$, we can see that the variability associated with presence is much higher than that with usage, which provides a side evidence that, for instance, occupants are found to be less likely to turn off their task lighting in case of temporary absence (Reinhart, 2004).

\subsection{Outcomes of the model of mean profiles and the variability model}

As an illustration, we show the comparison of mean occupancy profiles in Figure 12 and realized stochastic occupancy on a typical weekday in Figure 13. Since different levels in Table 1 could share the same mean profiles, we reorganize labels according to various mean profiles from all six levels in Figure 12. The correspondence to each level can be easily found in Table 1. 

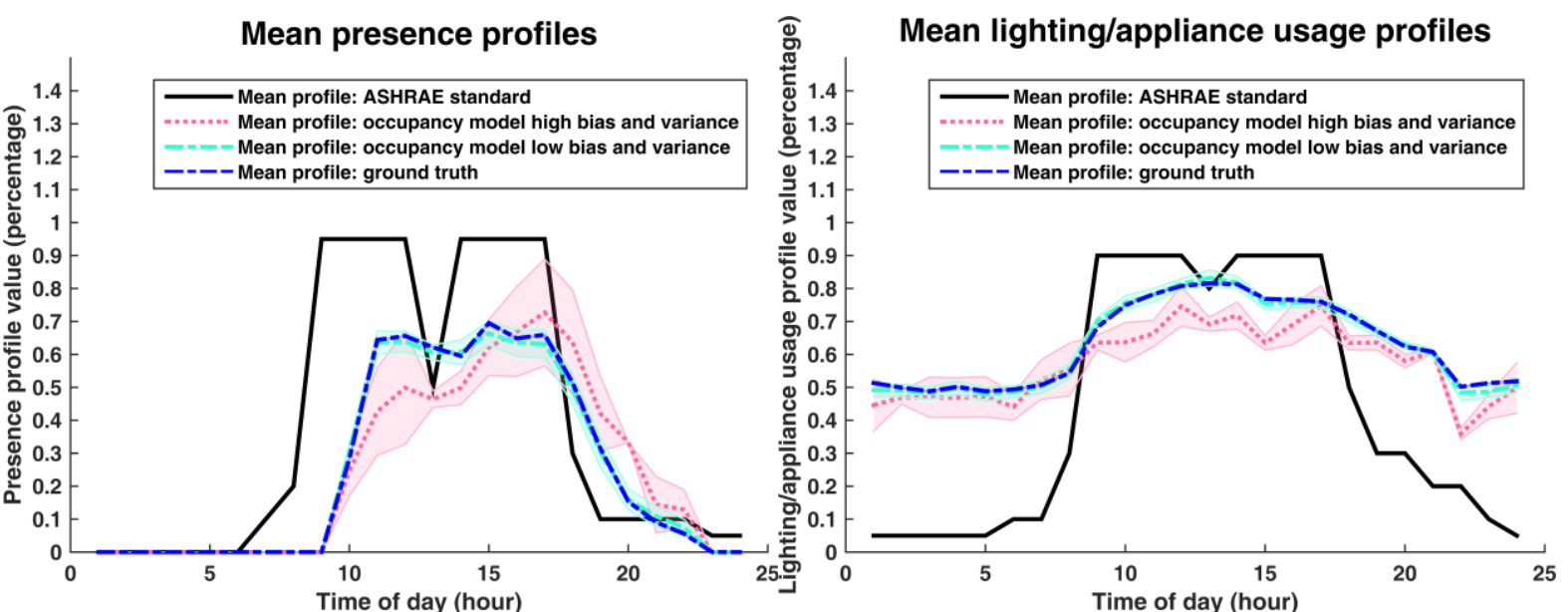

Figure 12 Mean occupancy profiles: presence profile (left) and lighting/appliance usage profile (right)
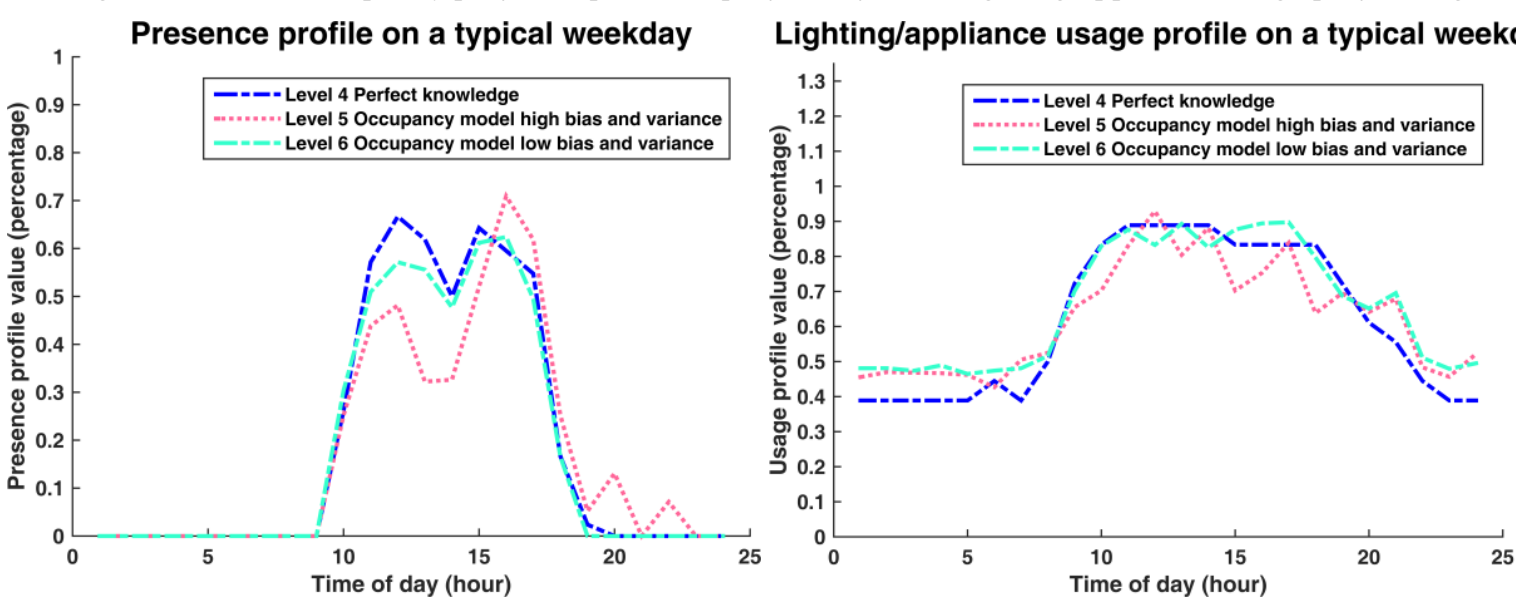

Figure 13 Presence profile (left) and lighting/appliance usage profile (right) on a typical weekday

Figure 12 highlights the large discrepancy between the ASHRAE standard profiles and mean profiles from the ground truth: the former greatly over-estimates the presence during the day while under-estimates the lighting/appliance usage during non-occupied hours. To be more specific, Level 1 profiles assume 9.2 personhours a day and 10.4 lighting/appliance-hours a day, while Level 4 profiles assume 5.9 person-hours a day and 15.1 lighting/appliance-hours a day. This indicates ignorant profiles for presence and lighting/appliance usage add up to $55 \%$ higher and $31 \%$ lower levels than the ones from the ground truth, respectively. Figure 12 also features a comparison between the two scenarios for the model of mean occupancy profiles, each with an added uncertainty band (reflecting the modeler's confidence in his estimate) around the mean. Indeed, profiles from the high bias and variance scenario deviate significantly from mean profiles from the ground truth, while those from the low bias and variance scenario closely follow the trend of actual occupancy (the green and blue curve virtually overlap). Furthermore, Figure 13 shows that a random realization generated by the low bias and variance scenario combined with the occupancy variability model can reproduce the characteristics of actual occupancy variables, while the other scenario introduces more inaccuracy.

\subsection{Direct energy outcomes}

500 For the study of the impact of occupancy variables on energy outcomes, we conduct uncertainty analysis with GURA-W (Georgia Tech Uncertainty and Risk Analysis Workbench) (B. D. Lee, Sun, Augenbroe, \& Paredis, 2013). We inject stochastic occupancy models into EnergyPlus simulations by manipulating the EnergyPlus IDF 
occupancy year, such that in each sample, the LHS sampler will randomly choose one column from the spreadsheet. For each level of occupancy information, 1000 LHS simulation runs are performed, and the resulting distribution is sometimes fitted with kernel density estimator (Kvam \& Vidakovic, 2007) for comparison.

Figure 14 compares the kernel density estimates for direct energy outcomes for the month of July in the medium office building in Atlanta. Recall that at Level 1 through 4, the lighting/appliance usage is not affected by the building energy model and is effectively deterministic, so usage at each level is shown as a vertical line in the figure. Since Level 2 through 4 all have the same mean usage in terms of lighting and appliances, corresponding lines overlap. If we relate these results back to Figure 12, discrepancy in daily lighting/appliance profiles have accumulated to monthly aggregates that show a clear disparity between Level 1 and Level 4 of about 23\% or $10161 \mathrm{kWh}$. This is the price to pay if we have no information available that may specialize the generic ASHRAE profiles. Furthermore, kernel density estimates in Level 5 and 6 demonstrate that inaccuracy in the modeler's estimate of lighting/appliance usage (Level 5) can lead to about $8 \%$ error against the ground truth in mean consumption and a significantly larger spread than the scenario with low bias and variance (Level 6).
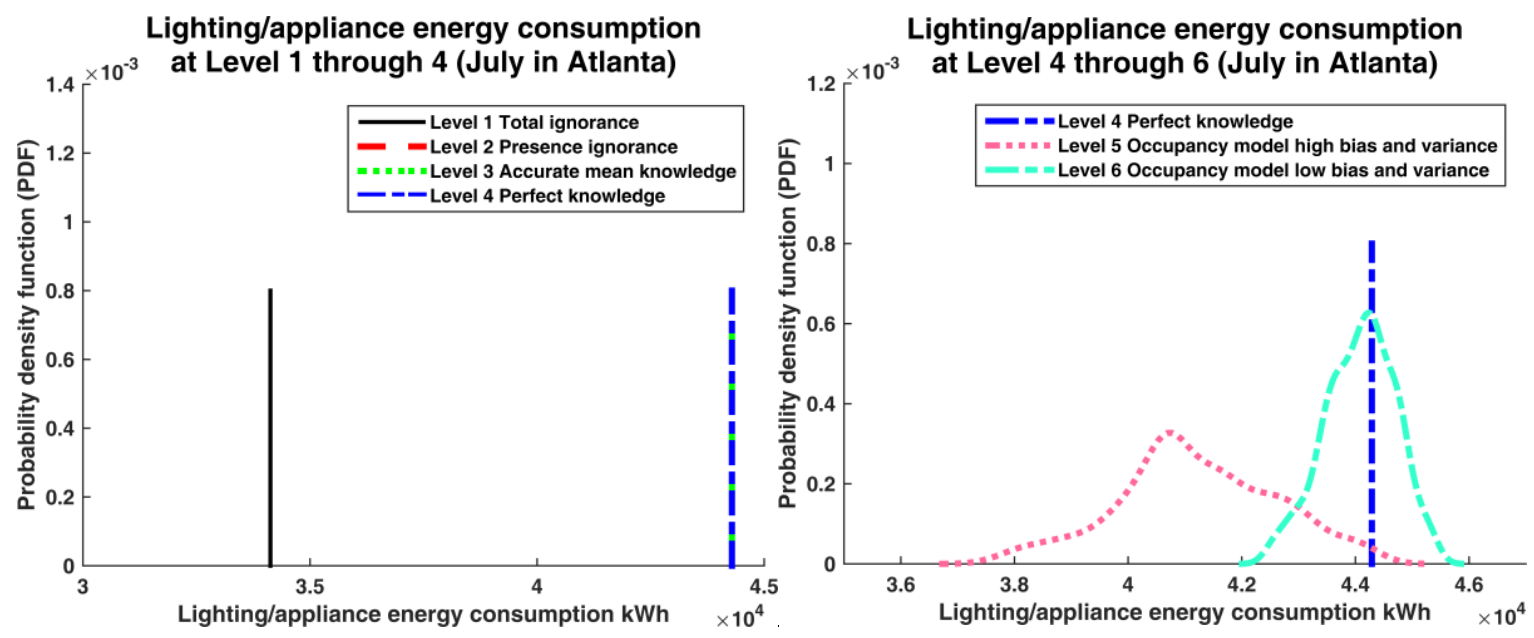

Figure 14 Distributions for direct energy consumption related to occupancy

\subsection{Indirect energy outcomes}

Figure 15 compares the kernel density estimates for aggregated indirect energy outcomes, including cooling energy consumption in the month of July and heating consumption in the month of January, taking into account the impact of occupancy models as well as all other sources of uncertainties as confounding factors. Results show that for the distribution of cooling and heating energy consumption, result of Level 3 is almost identical to that of Level 4, which suggests that perfect knowledge about the mean profiles of both occupancy variables is sufficient and the stochastic occupancy variability does not play a significant role for predicting aggregated energy consumption. Recall that ASHRAE standard profiles greatly over-estimate the mean presence while under-estimate the lighting/appliance usage, and that the comparison between Level 2 and 4 isolates the impact of presence, while the difference between Level 1 and 2 showcases the impact of lighting/appliance usage on indirect energy consumption. With this in mind, the results emphasize that given the design lighting/appliance and occupant presence density in the medium office building, the former has an evidently larger impact on aggregated indirect energy outcomes. Specifically, from Table 3 that summarizes the quantile values of these distributions, we observe by contrasting the mean of Level 2 and 4 that ignorant presence information alone, i.e. being $55 \%$ wrong with regard to presence, only translates to $2 \%$ error in the aggregated cooling energy and $3.6 \%$ 
in heating. For typical office buildings where internal loads are mainly attributed to lighting and appliances, such an observation suggests accuracy of the usage information should outweigh that of occupant presence information when it comes to aggregated energy outcomes. Furthermore, contrasting Level 5 and 6 with Level 4 showcases that apparent inaccuracy in the modeler's estimate of occupancy at Level 5 (Figure 12) only results in $4 \%$ error in the mean aggregated cooling energy and 5\% in heating against Level 4, which implies that building specific information, though subject to uncertainty and bias, seems preferable to generic ASHRAE assumptions
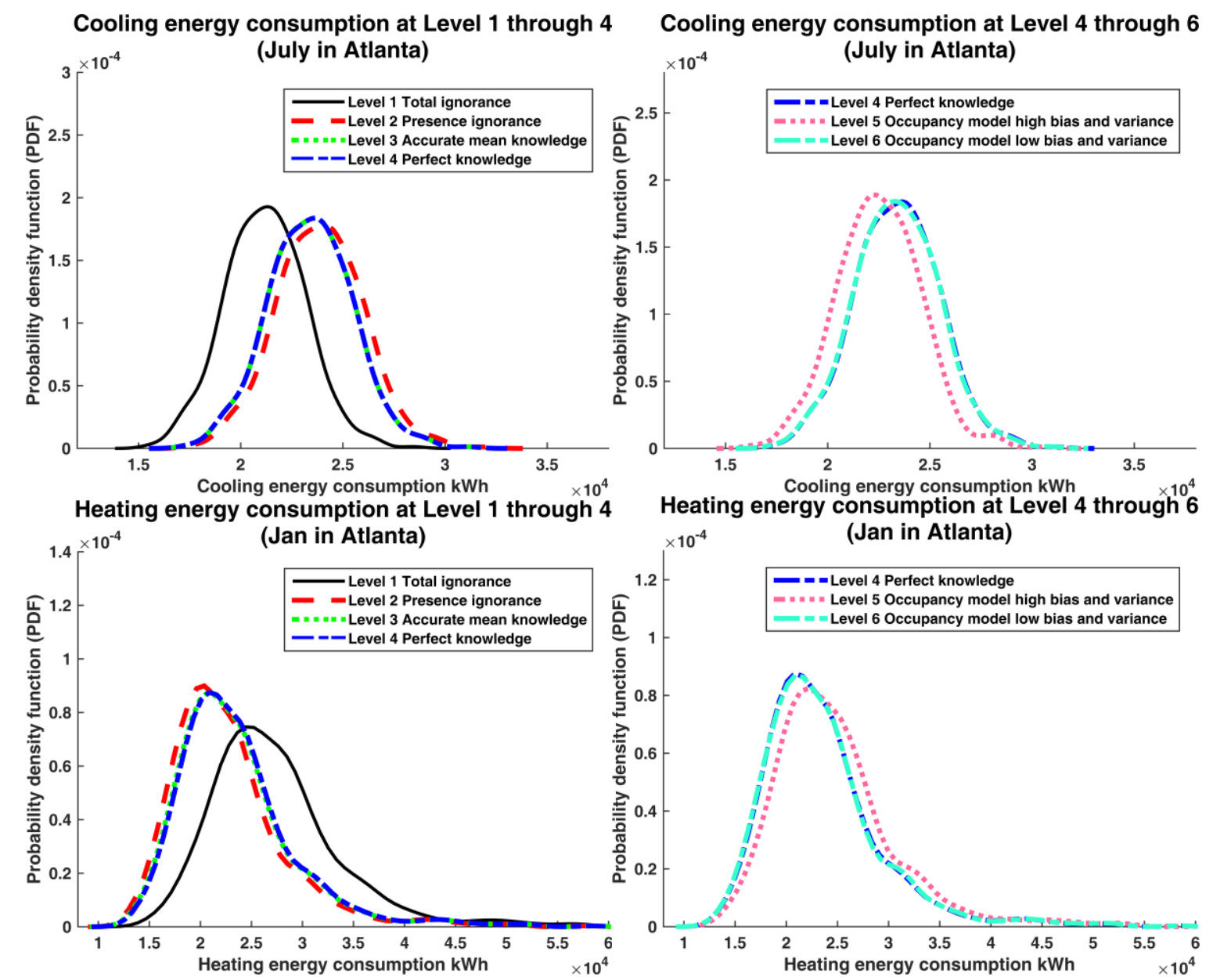

Figure 15 Distributions for indirect energy consumption related to occupancy: cooling energy in July (top row)

and heating energy in January (bottom row)

Table 3 Statistics of empirical distributions for indirect energy consumption at each Level

\begin{tabular}{|c|c|c|c|c|}
\hline \multicolumn{2}{|c|}{ Quantiles } & Mean & $10 \%$ & $90 \%$ \\
\hline \multirow{6}{*}{$\begin{array}{l}\text { Cooling } \\
\text { Energy } \\
\text { Consumption } \\
(\mathrm{kWh})\end{array}$} & Level 1 & 21197 & 18754 & 23628 \\
\hline & Level 2 & 23876 & 21269 & 26498 \\
\hline & Level 3 & 23411 & 20868 & 25949 \\
\hline & Level 4 & 23406 & 20855 & 25933 \\
\hline & Level 5 & 22504 & 19966 & 25021 \\
\hline & Level 6 & 23379 & 20811 & 25888 \\
\hline Heating & Level 1 & 27464 & 20575 & 35274 \\
\hline
\end{tabular}




\begin{tabular}{|c|c|c|c|c|}
\hline \multirow{5}{*}{$\begin{array}{l}\text { Energy } \\
\text { Consumption } \\
(\mathrm{kWh})\end{array}$} & Level 2 & 22824 & 16963 & 29749 \\
\hline & Level 3 & 23509 & 17489 & 30511 \\
\hline & Level 4 & 23672 & 17658 & 30661 \\
\hline & Level 5 & 24860 & 18613 & 32379 \\
\hline & Level 6 & 23656 & 17566 & 30930 \\
\hline
\end{tabular}

549 If aggregated energy consumption is the desired variable of interest, does simulation play a significant role in the 550 prediction of the impact of occupancy models on indirect energy outcomes? Can it be replaced with back-of-theenvelope calculations? Recall that Level 1 profiles assume 9.2 person-hours a day and 10.4 lighting/appliancehours a day, while Level 4 profiles assume 5.9 person-hours a day and 15.1 lighting/appliance-hours a day. Taking a stab at the above questions, we shift the distributions of monthly cooling consumption at Level 1 in July and April by a deterministic amount, determined by the difference between aggregated heat dissipation by occupants, lighting and appliances (Heat rate $\cdot$ Hour/Day · Day) at Level 1 and 4, divided by average cooling system $C O P$ :

Referring to the shifted new distribution as Level $1_{\text {aug }}$, we compare results at this level with those at Level 4 , shown in Figure 16. It is interesting to find out that the Level $1_{\text {aug }}$ distribution is very close to that produced with perfect occupancy information at Level 4 in July, but over-estimates in April. It appears to suggest that simulation is not needed to predict the impact of occupancy models on indirect energy outcomes in July, but that conclusion does not hold for April. This finding does not come as a surprise if one realizes that most of the extra heat dissipation from occupants, lighting and appliances leads directly to more cooling demand of the HVAC system in a cooling dominated month such as July, whereas in April the effect is not that easy to predict. Rather than always leading to more cooling load, during many hours in April, the extra heat increases the free-floating indoor temperature without reaching the cooling set-point and thus does not trigger cooling. Our simple hand calculation can obviously not predict this effect, and count the full amount of heat dissipation by occupants, lighting and appliances as an increase in the cooling demand, irrespective of month. The Level $1_{\text {aug }}$ distribution informed by the hand calculations will consequently over-estimate the impact of occupancy modeling in a swing month like April. Additionally, distributions at Level $1_{\text {aug }}$ and Level 4 also differ slightly in the spread of uncertainty, which is obviously attributable to nonlinear effects in the system, such as the impact of different occupancy levels on the part-load performance of the system. 

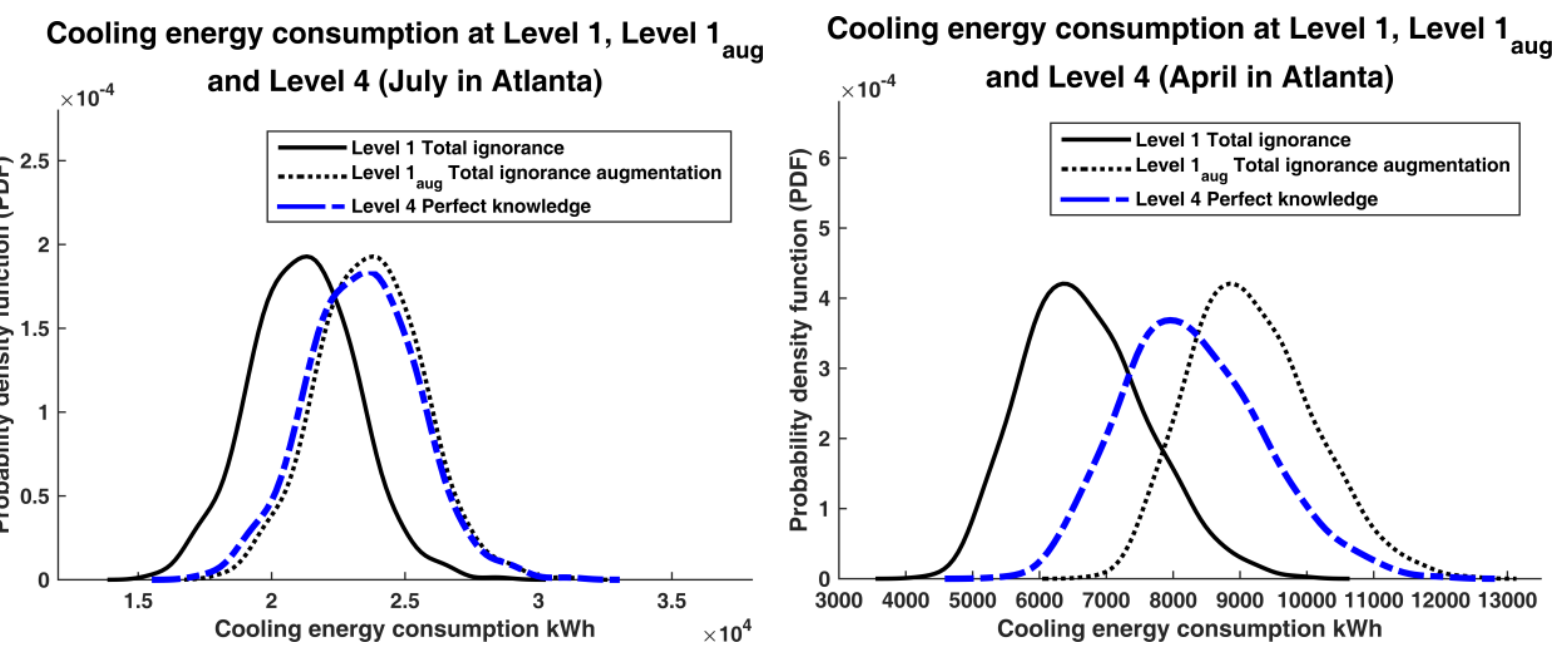

Figure 16 Discrepancy between Level 1, Level $1_{\text {aug }}$ and Level 4 for distributions of cooling energy consumption

in July (left) and April (right)

\subsection{Peak demand behavior}

All results thus far deal with aggregated energy outcomes. In order to inspect the peak demand behavior of the building, we generate power duration curves, which show the percentage of time over the period when building demand exceeds a certain power level as in Figure 17. Physical uncertainties other than occupancy contribute to the uncertainty band around the profile at each level. Except for Level 1, all other three profiles seem to be reasonably entwined, and this again shows the disadvantage of generic ASHRAE occupancy assumptions. However, after zooming into details and showing the percentage of time building power exceeds $175 \mathrm{~kW}$ (corresponding to area under the curve but above a line drawn horizontally at $175 \mathrm{~kW}$ on Figure 17), we find some remarkable results in Figure 18. All levels except for Level 6 fail to predict such behavior of the building. This result can be expected if one realizes that the ASHRAE standard profile assumes constant high occupancy every weekday starting at $9 \mathrm{am}$, which exaggerates the heavy usage of the building, leading to the peak of the empirical distribution in Level 1 heavily shifted to the right of Level 4. In contrast, in Level 2 and 3, average information on building occupancy without day-to-day fluctuations neglects heavily used hours of the building, and is therefore insufficient as well. Contrasting Level 5, 6 with Level 4 indicates that peak demand behavior of the building warrants the need for accurate occupancy information with low bias and uncertainty, as Level 5 performs as poorly as Level 2 and 3 and even Level 6 results differ slightly from the true distribution in Level 4 especially regarding the location of the maximum likelihood point. This is an instance where the energy modeler has to rely on stochastic occupancy modeling such as the one proposed in this paper, as well as on accurate knowledge about the typical occupancy of the building. 


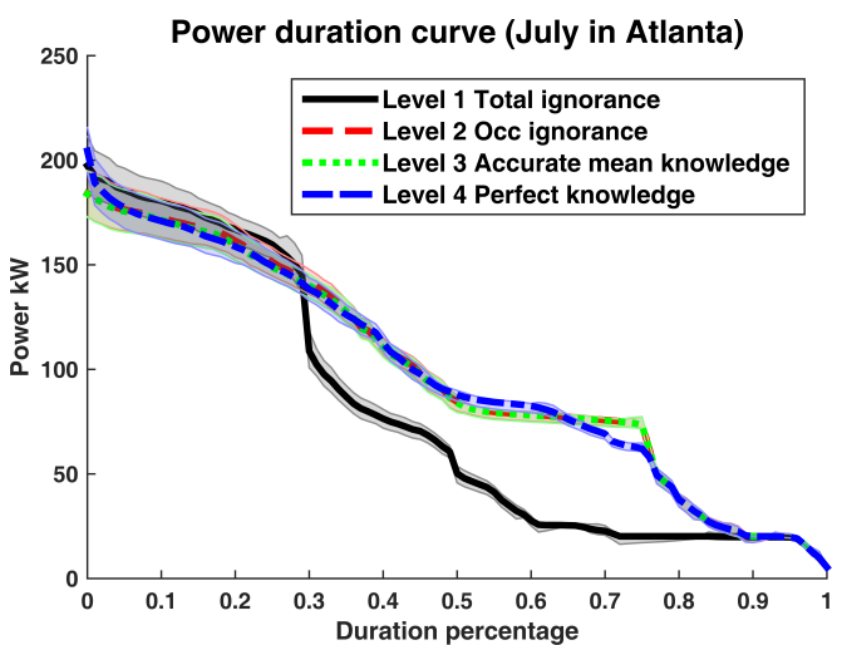

Figure 17 Power duration curves with uncertainty bands
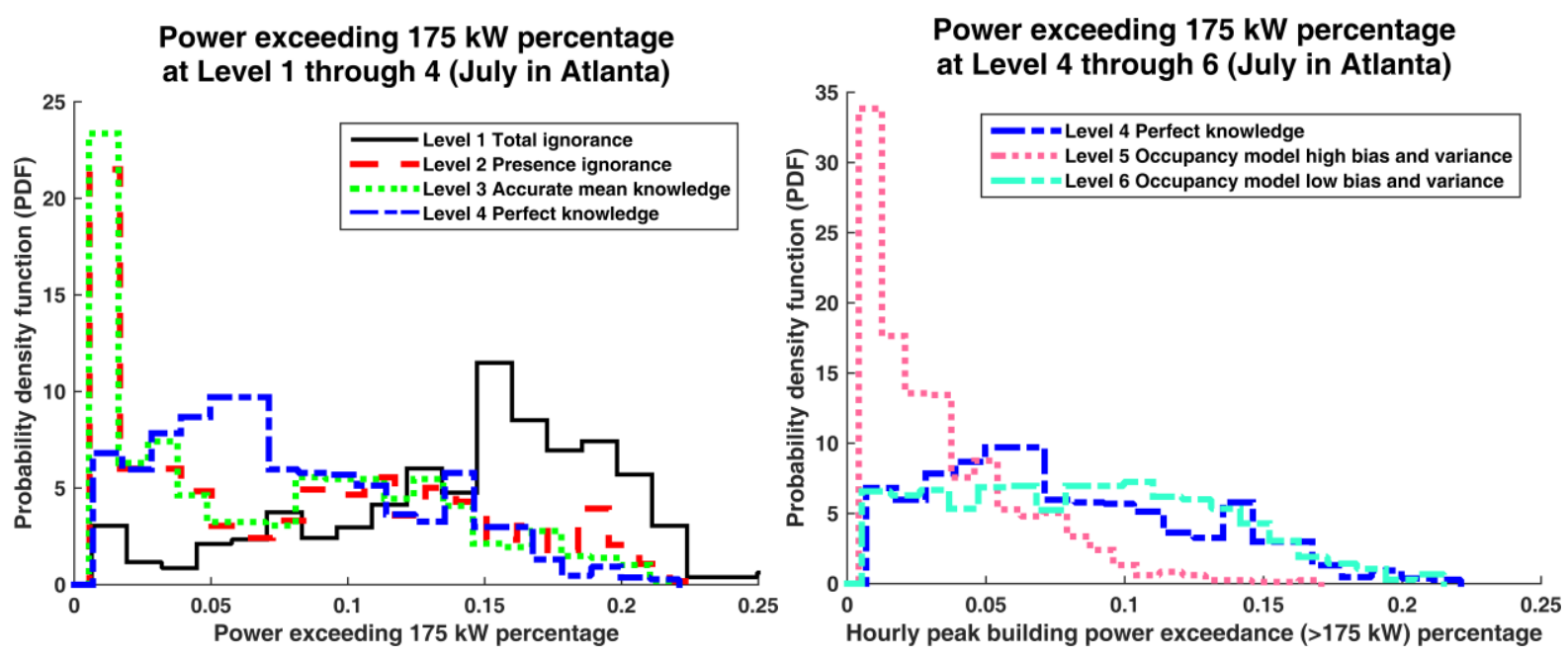

597

Figure 18 Percentage of time building power exceeds $175 \mathrm{~kW}$ in July in Atlanta

\subsection{Sensitivity index}

Last but not least, we show an index ranking in Figure 19 for parameters that show significant sensitivity to aggregated energy consumption in either the cooling or heating month. Note that at this final stage, we introduce two additional effects that we characterize with arbitrarily chosen uncertain ranges, i.e. without rigorous uncertainty quantification: thermostat control and spatial variability of occupant presence as described in Section 3.4. Additionally, for this sensitivity analysis, lighting/appliance usage is assumed to be accurately known (no uncertainty). A discrete parameter is introduced to reflect the model choice of temporal occupant presence from ignorant ASHRAE profiles to perfect knowledge. This parameter acts as a surrogate to rank the sensitivity of occupancy knowledge level amidst other regular sources of uncertainty. Regarding cooling energy consumption, six parameters combine to explain about $90 \%$ of response uncertainty, with the cooling set-point alone accounting for $36 \%$. The rest are related to the external convective heat transfer coefficient, infiltration, and ground reflectance and temperature. The model choice of temporal occupant presence barely makes it to the list, accounting for about $2.5 \%$ of outcome variance. Besides, spatial variability of occupant presence is shown to have no significant effect on cooling energy consumption. When it comes to heating energy consumption, it is expected that infiltration plays the major role, explaining $68 \%$ of response uncertainty, followed by external convective heat transfer coefficient, and the heating set-point. It is noteworthy that the heating set-point 
contributes a lot less to heating consumption uncertainty, than the cooling set-point to cooling consumption uncertainty. Again, the spatial variability of occupant presence plays a negligible role.

It should be well understood that these results are particular to the case study building, its HVAC system and the current control logic. The above methodology could be extended to other types of buildings with different systems and control logic. Such study would be useful to reveal which situations are particularly sensitive to the modeler's knowledge of occupancy. The case study seems to point towards the conclusion that the sensitivity of the indirect energy consumption to imprecise knowledge of occupancy variability is quite insignificant given the magnitude of many other sources of uncertainty.
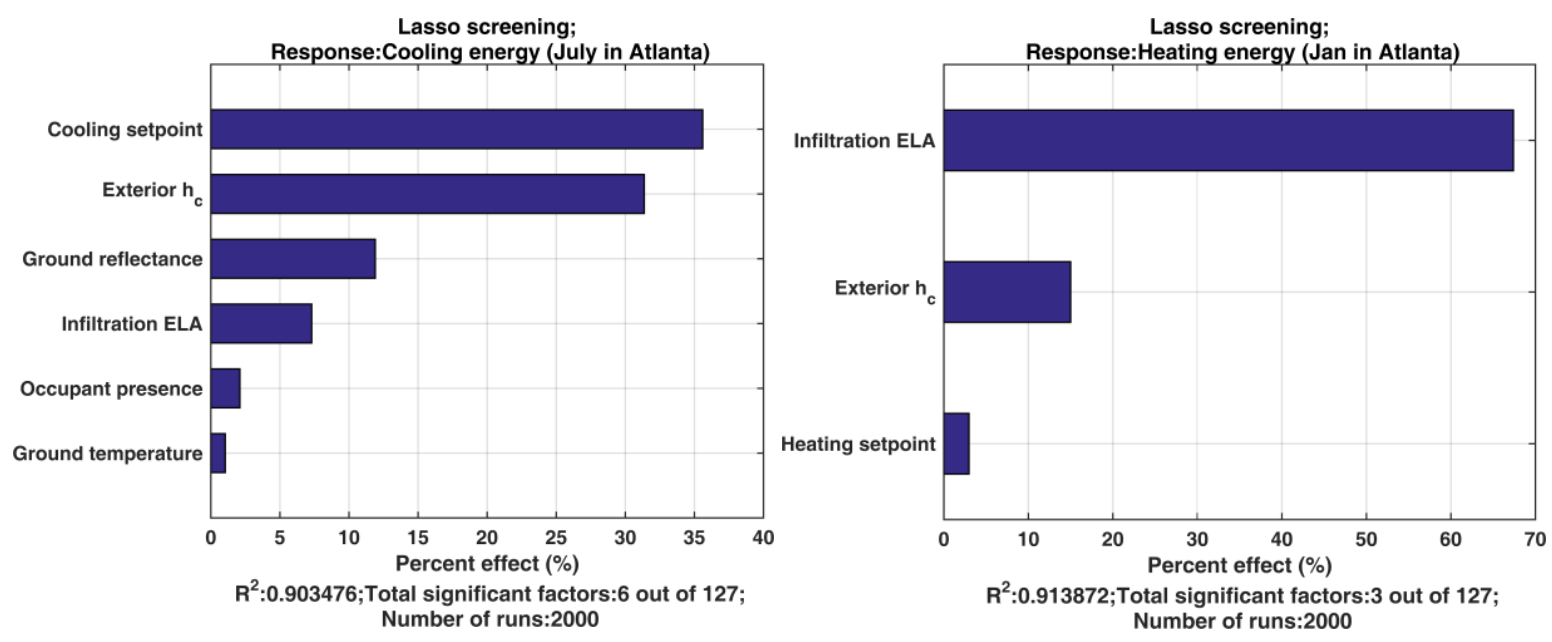

Figure 19 Sensitivity index ranking for significant uncertain parameters

\section{CONCLUSION}

We have proposed methods for modeling occupancy variables in office buildings: the multi-variant Gaussian distribution for the modeling of mean occupancy profiles and a hierarchical meta-model framework for occupancy variability that allows the interplay between two major occupancy variables with multi-dimensional time series techniques. A sensitivity analysis reveals the impact of different variants of occupancy models on outcomes of interest such as direct and indirect energy consumption, and peak demand behavior. The statistical modeling and dynamic simulations lead to the following conclusions:

(1) The mean occupancy can be modeled with the multi-variant Gaussian distribution, which accounts for the smoothness of mean occupancy profiles as well as the modeler's confidence in his estimate of typical building occupancy. Furthermore, occupancy variability can be represented by linear transfer function models. The derived models are able to generate stochastic occupancy profiles following the characteristics of the ground truth. Steps 2 and 3 of the proposed framework are generalizable to a future unfamiliar building, but they should undergo further testing and follow-up research.

(2) For aggregated building energy consumption, we argue for a clear separation between direct and indirect energy consumption and their individual sensitivity to occupancy variables. It proves that occupant presence has only a small impact on indirect and no impact on direct consumption, whereas lighting and appliance usage have evidently an immediate effect on direct and a moderate impact on indirect energy consumption. Specifically, being accumulatively $55 \%$ wrong with regard to presence, only translates to $2 \%$ error in aggregated cooling energy in July and 3.6\% error in heating energy in January. Such a finding redirects focus to improving the estimate of lighting and appliance usage, instead of that of presence, for a more 
accurate prediction of aggregate energy consumption. This is true in most common situations where the energy modeler estimates presence and usage separately or uses different approaches (e.g. survey for presence and data logging for lighting/appliance usage). Furthermore, it turns out that accurate mean knowledge is sufficient, that is, stochastic occupancy models do not play a significant role in the prediction of aggregated consumption in a conventional office building where the interaction between the operation of building systems and the spatial and temporal variability of occupancy is weak. More specifically, both the temporal and spatial variability in occupant presence drowns in light of all other uncertainties such as thermostat control and infiltration.

(3) For power duration curves that for instance describe the percentage of time building power exceeds a certain threshold, instances where building thermal peak demand coincides with occupancy peak matter. Therefore, a more accurate representation of occupancy with variability should be applied, whereas static profiles are inadequate.

We conclude with a reflection on the lessons that the results presented in this paper teach us. For aggregated consumption prediction in an office building, more efforts should be focused on the estimation of the mean profiles of lighting/appliance usage, as knowledge of presence is of much less importance for energy consumption outcomes. In both cases, a stochastic representation offers little benefit over mean profiles. If power duration curves are required, e.g. to predict the benefit of enrolling in a demand response program, we recommend practitioners apply the framework proposed in this paper for the stochastic representation of occupancy.

\section{ACKNOWLEDGEMENT}

This research was funded through the US NSF funded ERFI-SEED Award 1038248: Risk conscious design and retrofit for low energy (2010-2014), PI: Godfried Augenbroe, Georgia Institute of Technology, Atlanta, GA, USA.

\section{REFERENCES}

Abushakra, B., \& Claridge, D. E. (2008). Modeling Office Building Occupancy in Hourly Data-Driven and Detailed Energy Simulation Programs. ASHRAE Transactions, 114(2).

Abushakra, B., Sreshthaputra, A., Haberl, J. S., \& Claridge, D. E. (2001). Compilation of Diversity Factors and Schedules for Energy and Cooling Load Calculations, ASHRAE Research Project 1093-RP, Final Report: Energy Systems Laboratory, Texas A\&M University.

Aerts, D., Minnen, J., Glorieux, I., Wouters, I., \& Descamps, F. (2014). A Method for the Identification and Modelling of Realistic Domestic Occupancy Sequences for Building Energy Demand Simulations and Peer Comparison. Building and Environment, 75, 67-78. doi: 10.1016/j.buildenv.2014.01.021

Andersen, P. D., Iversen, A., Madsen, H., \& Rode, C. (2014). Dynamic Modeling of Presence of Occupants Using Inhomogeneous Markov chains. Energy and Buildings, 69, 213-223. doi: 10.1016/j.enbuild.2013.10.001

ASHRAE. (2004). 90.1 User's Manual ANSI/ASHRAE/IESNA Standard 90.1-2004 (pp. G-42). Atlanta, GA: The American Society of Heating Refrig-erating and Air Conditioning Engineers.

Azar, E., \& Menassa, C. C. (2012). Agent-Based Modeling of Occupants and Their Impact on Energy Use in Commercial Buildings. Journal of Computing in Civil Engineering, 26(4), 506-518. doi: $10.1061 /($ asce)cp.1943-5487.0000158

Bonte, M., Thellier, F., \& Lartigue, B. (2014). Impact of Occupant's Actions on Energy Building Performance and Thermal Sensation. Energy and Buildings, 76, 219-227. doi: 10.1016/j.enbuild.2014.02.068

Bronson, D. J., Hinchey, S. B., Haberl, J. S., \& O'Neal, D. L. (1992). Procedure for Calibrating the DOE-2 Simulation Program to Non-Weather-Dependent Measured Loads. Paper presented at the ASHRAE Winter Meeting, Anaheim, CA, USA, 01/25-29/92. 
Cali, D., Matthes, P., Huchtemann, K., Streblow, R., \& Muller, D. (2015). CO2 Based Occupancy Detection Algorithm: Experimental Analysis and Validation for Office and Residential Buildings. Building and Environment, 86, 39-49. doi: 10.1016/j.buildenv.2014.12.011

de Wit, S., \& Augenbroe, G. (2002). Analysis of Uncertainty in Building Design Evaluations and its Implications. Energy and Buildings, 34(9), 951-958. doi: 10.1016/s0378-7788(02)00070-1

Deru, M., Field, K., Studer, D., Benne, K., Griffith, B., Torcellini, P., . . Rosenberg, M. (2011). US Department of Energy Commercial Reference Building Models of the National Building Stock: National Renewable Energy Laboratory.

Duarte, C., Budwig, R., \& Van Den Wymelenberg, K. (2015). Energy and Demand Implication of Using Recommended Practice Occupancy Diversity Factors Compared to Real Occupancy Data in Whole Building Energy Simulation. Journal of Building Performance Simulation, 8(6), 408-423. doi: 10.1080/19401493.2014.966275

Dunn, G., \& Knight, I. (2005). Small Power Equipment Loads in UK Office Environments. Energy and Buildings, 37(1), 87-91. doi: 10.1016/j.enbuild.2004.05.007

Goyal, S., Barooah, P., \& Middelkoop, T. (2015). Experimental Study of Occupancy-Based Control of HVAC Zones. Applied Energy, 140, 75-84. doi: 10.1016/j.apenergy.2014.11.064

Haldi, F., \& Robinson, D. (2009). A Comprehensive Stochastic Model of Window Usage: Theory and Validation. Paper presented at the 11th International Building Performance Simulation Association Conference.

Heo, Y., Choudhary, R., \& Augenbroe, G. (2012). Calibration of Building Energy Models for Retrofit Analysis Under Uncertainty. Energy and Buildings, 47, 550-560. doi: 10.1016/j.enbuild.2011.12.029

Hopfe, C. J., \& Hensen, J. L. M. (2011). Uncertainty Analysis in Building Performance Simulation for Design Support. Energy and Buildings, 43(10), 2798-2805. doi: 10.1016/j.enbuild.2011.06.034

IEA-EBC Annex 66. (2015). Introduction. Retrieved from http://www.annex66.org/

Kvam, P. H., \& Vidakovic, B. (2007). Nonparametric Statistics with Applications to Science and Engineering (Vol. 653): John Wiley \& Sons.

Lee, B. D., Sun, Y., Augenbroe, G., \& Paredis, C. J. J. (2013). Toward Better Prediction of Building Performance: a Workbench to Analyze Uncertainty in Building Simulation. Paper presented at the 13th International Building Performance Simulation Association Conference, Chambéry, France.

Lee, Y. S., \& Malkawi, A. M. (2014). Simulating Multiple Occupant Behaviors in Buildings: An Agent-Based Modeling Approach. Energy and Buildings, 69, 407-416. doi: 10.1016/j.enbuild.2013.11.020

Li, N., Li, J. C., Fan, R. J., \& Jia, H. Y. (2015). Probability of Occupant Operation of Windows During Transition Seasons in Office Buildings. Renewable Energy, 73, 84-91. doi: 10.1016/j.renene.2014.05.065

Liao, C. D., Lin, Y. S., \& Barooah, P. (2012). Agent-Based and Graphical Modelling of Building Occupancy. Journal of Building Performance Simulation, 5(1), 5-25. doi: 10.1080/19401493.2010.531143

Lindelof, D., \& Morel, N. (2006). A Field Investigation of the Intermediate Light Switching by Users. Energy and Buildings, 38(7), 790-801. doi: 10.1016/j.enbuild.2006.03.003

Lunn, D., Jackson, C., Best, N., Thomas, A., \& Spiegelhalter, D. (2012). The BUGS Book: A Practical Introduction to Bayesian Analysis: CRC press.

Macdonald, I., \& Strachan, P. (2001). Practical Application of Uncertainty Analysis. Energy and Buildings, 33(3), 219-227. doi: 10.1016/s0378-7788(00)00085-2

Mahdavi, A. (2010). People in Building Performance Simulation. In J. Hensen \& R. Lamberts (Eds.), Building Performance Simulation for Design and Operation (pp. 56-83).

Menezes, A. C., Cripps, A., Bouchlaghem, D., \& Buswell, R. (2012). Predicted vs. Actual Energy Performance of Non-domestic Buildings: Using Post-Occupancy Evaluation Data to Reduce the Performance Gap. Applied Energy, 97, 355-364. doi: 10.1016/j.apenergy.2011.11.075

O'Hagan, A., Buck, C. E., Daneshkhah, A., Eiser, J. R., Garthwaite, P. H., Jenkinson, D. J., . . Rakow, T. (2006). Uncertain Judgements: Eliciting Experts' Probabilities: John Wiley \& Sons.

Oldewurtel, F., Sturzenegger, D., \& Morani, M. (2013). Importance of Occupancy Information for Building Climate Control. Applied Energy, 101, 521-532. doi: 10.1016/j.apenergy.2012.06.014

Page, J., Robinson, D., Morel, N., \& Scartezzini, J. L. (2008). A Generalised Stochastic Model for the Simulation of Occupant Presence. Energy and Buildings, 40(2), 83-98. doi: 10.1016/j.enbuild.2007.01.018

Parys, W., Saelens, D., \& Hens, H. (2011). Coupling of Dynamic Building Simulation with Stochastic Modelling of Occupant Behaviour in Offices - a Review-Based Integrated Methodology. Journal of Building Performance Simulation, 4(4), 339-358. doi: 10.1080/19401493.2010.524711

Reinhart, C. F. (2004). Lightswitch-2002: a Model for Manual and Automated Control of Electric Lighting and Blinds. Solar Energy, 77(1), 15-28. doi: 10.1016/j.solener.2004.04.003 
Roberson, J. A., Webber, C. A., McWhinney, M. C., Brown, R. E., Pinckard, M. J., \& Busch, J. F. (2004). AfterHours Power Status of Office Equipment and Inventory of Miscellaneous Plug-Load Equipment. Lawrence Berkeley National Laboratory.

Rosenblum, B. (2012). Collecting Occupant Presence Data for Use in Energy Management of Commercial Buildings (Master Thesis). University of California, Berkeley. Retrieved from http://escholarship.org/uc/item/1pz2528w

Rysanek, A. M., \& Choudhary, R. (2015). DELORES - an Open-Source Tool for Stochastic Prediction of Occupant Services Demand. Journal of Building Performance Simulation, 8(2), 97-118. doi: $10.1080 / 19401493.2014 .888595$

Saelens, D., Parys, W., \& Baetens, R. (2011). Energy and Comfort Performance of Thermally Activated Building Systems Including Occupant Behavior. Building and Environment, 46(4), 835-848. doi: 10.1016/j.buildenv.2010.10.012

Schweigler, L. M., Desmond, J. S., McCarthy, M. L., Bukowski, K. J., Ionides, E. L., \& Younger, J. G. (2009). Forecasting Models of Emergency Department Crowding. Academic Emergency Medicine, 16(4), 301308. doi: 10.1111/j.1553-2712.2009.00356.x

Shih, H. C. (2014). A Robust Cccupancy Detection and Tracking Algorithm for the Automatic Monitoring and Commissioning of a Building. Energy and Buildings, 77, 270-280. doi: 10.1016/j.enbuild.2014.03.069

Sun, Y. (2014). Closing the Building Energy Performance Gap by Improving Our Predictions (Doctoral Dissertation). Retrieved from http://hdl.handle.net/1853/52285 Georgia Tech Theses and Dissertations database.

Sun, Y., Gu, L., Wu, C. J., \& Augenbroe, G. (2014). Exploring HVAC System Sizing Under Uncertainty. Energy and Buildings, 81, 243-252.

Tabak, V. (2008). User Simulation of Space Utilisation: Eindhoven University Press.

Tahmasebi, F., \& Mahdavi, A. (2015). The Sensitivity of Building Performance Simulation Results to the Choice of Occupants' Presence Models: A Case Study. Journal of Building Performance Simulation, 1-11. doi: 10.1080/19401493.2015.1117528

Tian, W. (2013). A Review of Sensitivity Analysis Methods in Building Energy Analysis. Renewable and Sustainable Energy Reviews, 20, 411-419. doi: http://dx.doi.org/10.1016/j.rser.2012.12.014

Tibshirani, R. (1996). Regression Shrinkage and Selection via the Lasso. Journal of the Royal Statistical Society. Series B (Methodological), 267-288.

Wang, C., Yan, D., \& Jiang, Y. (2011). A Novel Approach for Building Occupancy Simulation. Building Simulation, 4(2), 149-167. doi: 10.1007/s12273-011-0044-5

Wang, L. P., Mathew, P., \& Pang, X. F. (2012). Uncertainties in Energy Consumption Introduced by Building Operations and Weather for a Medium-Size Office Building. Energy and Buildings, 53, 152-158. doi: 10.1016/j.enbuild.2012.06.017

Widen, J., Lundh, M., Vassileva, I., Dahlquist, E., Ellegard, K., \& Wackelgard, E. (2009). Constructing Load Profiles for Household Eectricity and Hot Water from Time-Use Data-Modelling Approach and Validation. Energy and Buildings, 41(7), 753-768. doi: 10.1016/j.enbuild.2009.02.013

Zhao, J., Lasternas, B., Lam, K. P., Yun, R., \& Loftness, V. (2014). Occupant Behavior and Schedule Modeling for Building Energy Simulation Through Office Appliance Power Consumption Data Mining. Energy and Buildings, 82, 341-355. doi: 10.1016/j.enbuild.2014.07.033 